\title{
Transcriptional regulation of gene expression clusters in motor neurons following spinal cord injury
}

\author{
Jesper Ryge ${ }^{* 1}$, Ole Winther2,3, Jacob Wienecke4, Albin Sandelin², Ann-Charlotte Westerdahl1', Hans Hultborn ${ }^{4}$ and \\ Ole Kiehn*1
}

\begin{abstract}
Background: Spinal cord injury leads to neurological dysfunctions affecting the motor, sensory as well as the autonomic systems. Increased excitability of motor neurons has been implicated in injury-induced spasticity, where the reappearance of self-sustained plateau potentials in the absence of modulatory inputs from the brain correlates with the development of spasticity.

Results: Here we examine the dynamic transcriptional response of motor neurons to spinal cord injury as it evolves over time to unravel common gene expression patterns and their underlying regulatory mechanisms. For this we use a rat-tail-model with complete spinal cord transection causing injury-induced spasticity, where gene expression profiles are obtained from labeled motor neurons extracted with laser microdissection 0, 2, 7, 21 and 60 days post injury. Consensus clustering identifies 12 gene clusters with distinct time expression profiles. Analysis of these gene clusters identifies early immunological/inflammatory and late developmental responses as well as a regulation of genes relating to neuron excitability that support the development of motor neuron hyper-excitability and the reappearance of plateau potentials in the late phase of the injury response. Transcription factor motif analysis identifies differentially expressed transcription factors involved in the regulation of each gene cluster, shaping the expression of the identified biological processes and their associated genes underlying the changes in motor neuron excitability.

Conclusions: This analysis provides important clues to the underlying mechanisms of transcriptional regulation responsible for the increased excitability observed in motor neurons in the late chronic phase of spinal cord injury suggesting alternative targets for treatment of spinal cord injury. Several transcription factors were identified as potential regulators of gene clusters containing elements related to motor neuron hyper-excitability, the manipulation of which potentially could be used to alter the transcriptional response to prevent the motor neurons from entering a state of hyper-excitability.
\end{abstract}

\section{Background}

The mammalian central nervous system has limited capability for regeneration. Spinal cord injury therefore leads to neurological dysfunctions affecting the motor, sensory as well as the autonomic systems [1]. In the immediate phase following spinal cord injury the excitability of the motor networks caudal to the injury becomes depressed. This initial state of motor depression is often followed by a maladaptive increase in network excitability resulting in

* Correspondence: jesper.ryge@epfl.ch, O.Kiehn@ki.se

Mammalian Locomotor Laboratory, Department of Neuroscience, Karolinska Institutet, Retzius väg 8, 17177 Stockholm, Sweden

Full list of author information is available at the end of the article spasticity and/or pain [2-6]. The injury-induced spasticity is characterized by a disturbing hyper-reflexia causing prolonged muscle activity upon short activation of sensory afferents $[7,8]$. Increased excitability of spinal motor neurons, the cells that transduce the reflex response to the muscles, has been implicated in this pathophysiological state.

Under normal physiological conditions the motor output (gain) can be modulated by activation of channels in the motor neurons that conduct persistent inward currents, resulting in plateau potentials and sustained firing, leading to enhanced and prolonged muscle contraction 
[9-14]. The expression of plateau potentials depends on metabotropic receptor activation including activation of noradrenergic and/or serotonergic receptors. The neuromodulators that activate these receptors primarily originate from neurons located in the brainstem, which project descending fibers to the spinal cord. The ability to generate plateau potentials therefore disappears in motor neurons located caudal to a spinal cord injury [10,15-21]. They spontaneously reappear two to three weeks after injury due to chronic changes in motor neuron properties that parallel development of injury-induced spasticity [22-24]. To investigate the molecular mechanisms underlying the reappearance of plateau potentials after spinal cord injury we recently undertook a global gene expression study of motor neurons in the late phase of injuryinduced spasticity [25] using the rat-tail-model with a complete spinal cord transection at the S2 segment, developed by Bennett and coworkers [6]. This work identified differential expression of genes relating to ion channels, neurotransmitter receptors and intracellular pathways 21 and 60 days post injury, supporting the observed increase in motor neuron excitability and the reappearance of plateau potentials [25]. In the present work we investigate the dynamic transcriptional response of motor neurons following spinal cord injury $0,2,7,21$ and 60 days post injury, enabling us to dissect out some of the regulatory mechanisms of transcription underlying the observed hyper-excitability. In the brain, such dynamic transcriptome analyses have been used to analyze the gene expression pattern of well-defined cell populations during development [26,27]. Comprehensive studies on the mechanisms of transcriptional regulation have mostly been conducted on simpler model systems with homogenous cell populations such as cell cultures [28-31]. In the present study the transcriptional response of motor neurons over time constitute a direct measure of cell-specific processes in a complex anatomical structure, allowing us in a similar fashion to examine the expression patterns and the underlying regulatory mechanisms of this response.

Cluster analysis of the gene expression time series identifies 12 time profiles reflecting combinations of early and late transcriptional regulations. Ontology analysis shows that these clusters contain groups of genes that define over-represented ontologies, indicating that each cluster profile reflects the timing of distinct biological processes as the motor neurons respond to the injury. Genes previously implicated in the development of the plateau potentials in injury-induced spasticity [32] are also identified as differentially expressed over time. The general injury response is paralleled by a response in the regulatory networks of transcription factors. Transcription factor motif analysis of the gene promoter sequences belonging to each time profile indicates a complex regulatory control of the different time profiles. Such transcription factors could prove to be potential targets for treatment of injury-induced spasticity as well as other aspects of the injury response, where experimental manipulation of their expression could be used to alter the transcriptional response of motor neurons preventing them from entering a state of hyper-excitability.

\section{Results}

\section{Transcriptional response of motor neurons to injury}

Spinal cord injury was inflicted by a complete spinal cord transection at the second sacral segment (S2), in effect disconnecting the spinal networks caudal to the lesion from the remaining part of the central nervous system. The injury causes complete paralysis of the tail, with no effect on bladder, bowel or hind limb functions [6,32]. The motor paralysis of the tail is followed by a slowly developing spasticity [33] in the weeks and months after injury. Clinical as well as electrophysiological evaluation of tail spasticity was performed at each time point, showing a progressive development of spasticity (or hyperreflexia) saturating between 21 and 60 days post injury (Figure 2 in [32]).

To examine and compare the transcriptional response of the motor neurons in early and late post-injury phases GeneChip Rat Genome 230_2.0 Arrays (Affymetrix, RAT230 2 chip) were hybridized with RNA samples originating from motor neurons of uninjured control animals $(\mathrm{n}=4)$ as well as animals $2(\mathrm{n}=6), 7(\mathrm{n}=5), 21(\mathrm{n}=8)$ and $60(\mathrm{n}=8)$ days post injury. A conglomerate classifier based on three well-established adjusted ANOVA teststatistics for microarray analysis (limma, Cyber-T and SAM) was used to identify significantly differentially expressed genes used for subsequent clustering, identifying 3,708 genes with a set false discovery rate (FDR) threshold of 0.02 [34].

\section{Consensus clustering unravels distinct gene expression time profiles}

In order to identify common expression profiles across time among the differentially expressed genes, transcripts were grouped into clusters of similar expression patterns using a robust consensus cluster algorithm developed by Grotkjaer et al. 2006 [35]. The consensus cluster algorithm is based on an averaging procedure conducted on multiple runs of K-means clustering (see "Methods"). This procedure amplifies common patterns in the expression profiles while suppressing non-reproducible features. To reduce miss-classification due to noise in the expression data (of non-differentially expressed genes) we use the consensus clustering on the most likely differentially expressed genes. With a 0.02 FDR level of significance 3,708 probe sets were included in the consensus cluster analysis. The analysis revealed the existence of 12 distinct 
A

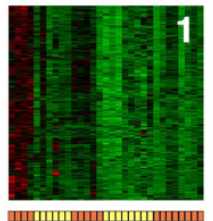

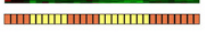

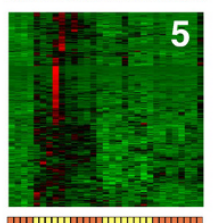

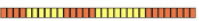

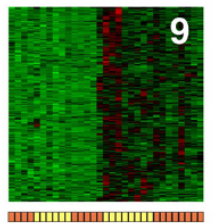

$\begin{array}{lllll}0 & 2 & 7 & 21 & 60\end{array}$

B
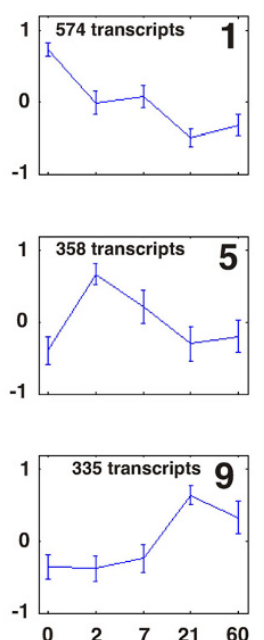

C

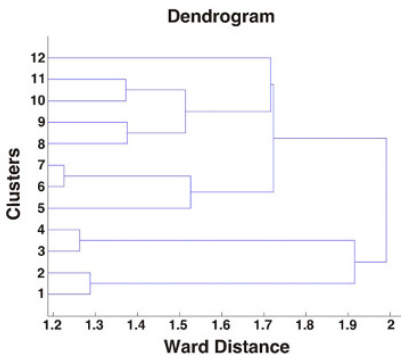

Heatmaps of Consensus Clusters
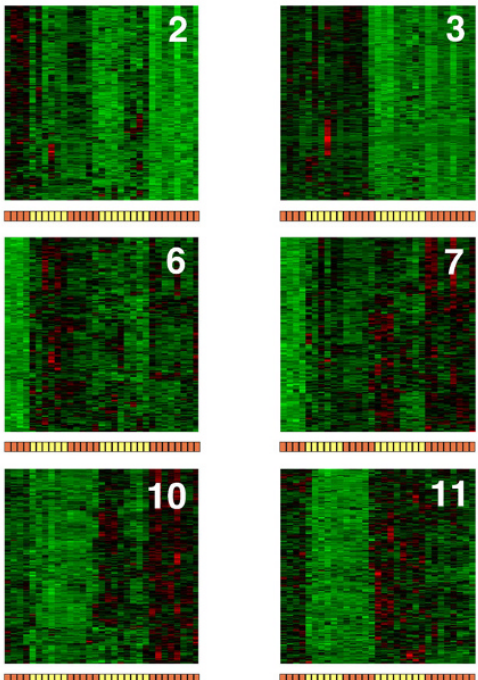

$\begin{array}{llll}0 & 5 & 21 & 0\end{array}$

$\begin{array}{lllll}0 & 2 & 7 & 21 & 60\end{array}$

Time Profiles of consensus clusters
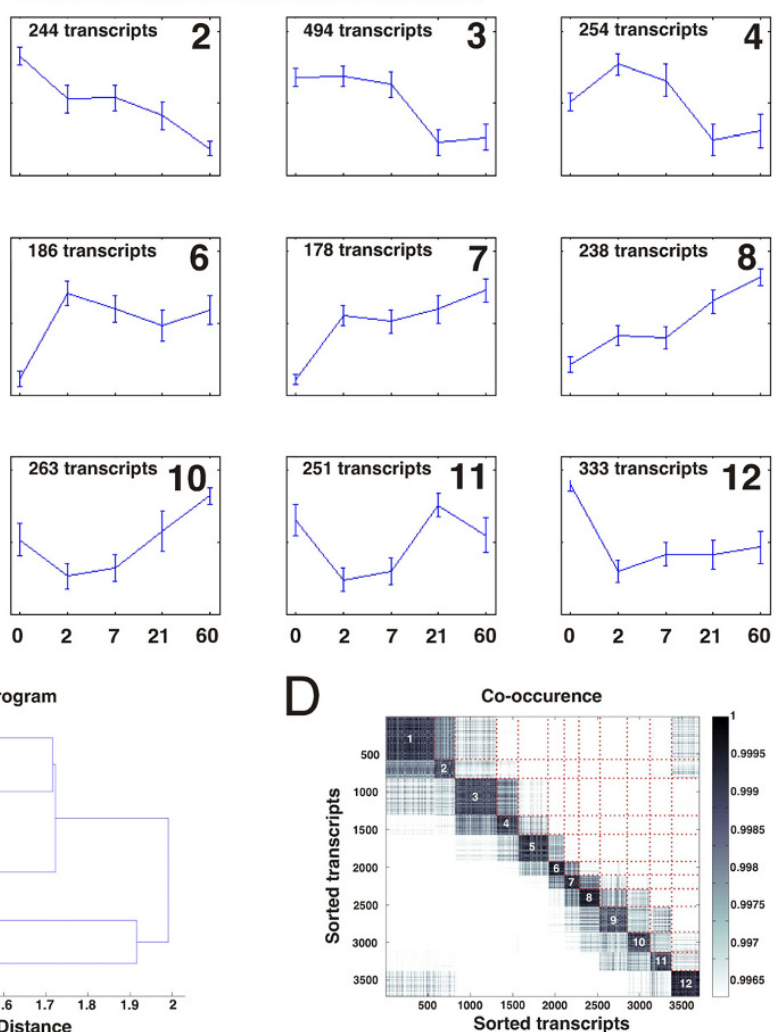

Figure 1 Consensus clustering define distinct time profiles of gene expression. Consensus clusters of the 3,708 most differentially expressed genes. A. Heatmaps of each consensus cluster illustrating the expression pattern of the genes contained in each cluster. The gene wise expression has been normalized according to eq. 1 resulting in expression between -1 and +1 . Color Code: Red signifies up-regulation and green down-regulation compared to the gene-wise average (see color bar). The alternating colored bars below each heatmap illustrate the microarrays of each time point $0,2,7,21$ and 60 days post injury. B. The average expression time profile of each consensus cluster, plus/minus one standard deviation. C. Optimal leaf ordered dendrogram showing the Ward distance between each consensus cluster. D. Contour map of the (leaf ordered) co-occurrence matrix. The consensus clusters are indicated along the diagonal with numbers. This figure illustrates the gene overlap between clusters for the consecutive cluster runs with randomized initial settings. 
time profiles (Figure 1A-1B) each containing 178-574 genes. Increasing the amount of consensus clusters beyond 12 did not reveal new patterns of expressions but rather breaks down the existing time profiles into subcategories with very similar features. Heatmaps of each of the 12 consensus clusters, Figure 1A, show how their constituent genes change expression over time across all the microarrays in the study, where the expression levels for each transcript have been centered and normalized according to eq 1. Red color signifying expression above the average for a given transcript and green color signifies below average (see color bar). For each cluster the average level of (normalized) expression across all its constituent genes was calculated for each time point producing cluster-specific time profiles of expression, shown in Figure $1 \mathrm{~B}$ - in the following termed cluster profiles. The similarity between cluster profiles is illustrated by the dendrogram of Figure $1 \mathrm{C}$ as well as in the contour map of the optimal leaf ordered co-occurrence matrix shown in Figure 1D. The co-occurrence contour map illustrates the degrees of gene overlap between the clusters in all the Kmeans cluster runs, where well defined squares represents very robust clusters while points falling outside these areas represents genes that occasionally fall into other clusters. The co-occurrence contour map is fairly well defined along the diagonal where overlapping genes for the most part are limited to neighboring clusters.

The expression time profiles fall into two main branches in the dendrogram, with profiles 1-4 in one branch and profiles 5-12 in the other. The first group (profiles 1-4) shares a pattern of late (21 and 60 days) down-regulation, whereas their early response varies $(2$ and 7 days). In the other group (profiles 5-12), all but profile 12 are characterized by an up-regulation either early (profile 5), late (profiles 8-10) or both (profiles 6-7). Profiles 10 and 11 have an early down-regulation followed by a subsequent up-regulation. Cluster profile 12 is characterized by a very substantial early down-regulation followed by a slight progressive increase in expression that remains below control levels. This makes it somewhat similar to cluster profiles 10 and 11, though as it remains below control levels even in the late phase its biological classification rather belongs to the group of down-regulated cluster profiles 1-4. The co-occurrence plot, Figure 1D, also illustrates this fact, where genes of profile 12 occasionally overlap with cluster profiles 1 and 2 as well as with cluster profiles 10 and 11 . The full list of differentially expressed genes grouped according to their cluster identification is provided in Additional file 1.

The consensus clustering of the differentially expressed genes thus unravels gene clusters with distinct expression time profiles. We used these clusters for ontology analysis as well as to ascribe expression profiles to genes related to motor neuron excitability changes. Analysis of transcrip- tion factor regulation was performed on each cluster to identify potential mechanisms of regulation for the identified genes and ontologies.

\section{Ontology analysis of gene expression time profiles}

Genes may be annotated according to well-defined ontologies such as biological processes, cellular location, biochemical pathways, protein families etc. highlighting different aspect of their function. Over-representation of genes within a cluster profile that share an ontology term strongly suggests that the cluster represents a set of genes that engage in or represent the identified ontology [36]. The ontology database maintained by the gene ontology (GO) consortium annotating genes according to biological processes (BP), molecular functions (MF) and cellular compartments $(\mathrm{CC})$ as well as INTERPRO and KEGG pathways were all used in this analysis. Each ontology highlights a different aspect of gene functions, making it desirable to group together over-represented ontologies containing a predefined degree of gene overlap in order to fully appreciate the functional role of each of the 12 time profiles. Furthermore, since gene ontologies are structured as acyclic directed graphs where a gene is annotated from its most descriptive (lowest) level all the way up through the graph, it may also be desirable to group together the annotation terms within each branch in the ontology graph that share the same genes and within these extract the most representative ontology (the lowest level) to exclude redundant representations. DAVID functional ontology clustering accomplishes this task. The ontology analysis was run on each time profile with the total set of differentially expressed genes as background. The most representative term of each ontology cluster with a p-value below 0.03 was extracted, shown in Table 1. It is clear from this analysis that profiles 1-4 and 12, all signified by a late down-regulation, have several overlapping terms. Among the over-represented ontologies defined by the down-regulated genes contained in each of these clusters, we find "cell-cell adhesion" and "zinc finger" shared between profiles 1 and 3, ontologies relating to ribosomal processes shared in profiles 2 and 3, and ontologies relating to metabolic processes shared in profiles 3 and 4 . Profile 12 contains genes that seem to be involved in mitochondrial energy production (ATP) and regulation of anion concentrations and signaling. There are some terms in cluster profiles 1-4 that also seem to indicate a down-regulation of mitochondrial processes and overall metabolism. The profiles signified by an up-regulation of transcripts somewhere along the time profile (cluster profiles 5-11) engage in different processes from those observed for cluster profiles $1-4$ and 12 . Profile 5 represents an early response of upregulated genes primarily seen two days post injury involving processes of immunological and inflammatory 
Table 1: Over-represented ontologies of each gene cluster

\begin{tabular}{llrrr}
\hline Ontology Term & Class & Count & Total & Terms \\
\hline Cluster 1: 574 transcripts & & & \\
& & & \\
\hline Cell-cell adhesion & BP & 18 & 34 & 9 \\
Ensheathment of neurons & BP & 7 & 7 & 11 \\
Zinc finger, C2H2-type & INTERPRO & 7 & 9 \\
L-amino acid transmembrane transporter activity & MF & 6 & 9 \\
Solute:cation symporter activity & MF & 6 & 11 & 10
\end{tabular}

Cluster 2: 244 transcripts

$\begin{array}{llrrr}\text { Ribonucleoprotein complex } & \text { CC } & 31 & 54 & 16 \\ \text { Translation } & \text { BP } & 23 & 54 & 16 \\ \text { Intracellular part } & \text { CC } & 143 & 162 & 8 \\ \text { RNA splicing } & \text { BP } & 9 & 14 & 9 \\ \text { Ribonucleoprotein complex biogenesis } & \text { BP } & 10 & 14 & 4 \\ \text { Macromolecule metabolic process } & \text { BP } & 95 & 110 & 4 \\ \text { Mitochondrial ribosome } & \text { CC } & 4 & 4 \\ \text { Regulation of apoptosis } & \text { BP } & 16 & 37\end{array}$

Cluster 3: 494 transcripts

\begin{tabular}{|c|c|c|c|c|}
\hline Cell-cell adhesion & $\mathrm{BP}$ & 22 & 44 & 11 \\
\hline Metal ion binding & MF & 97 & 98 & 4 \\
\hline Cellular metabolic process & $\mathrm{BP}$ & 198 & 217 & 4 \\
\hline Regulation of biosynthetic process & $\mathrm{BP}$ & 12 & 19 & 4 \\
\hline Macromolecule biosynthetic process & $\mathrm{BP}$ & 37 & 52 & 4 \\
\hline Ribosome & KEGG & 12 & 38 & 9 \\
\hline Cell part & $\mathrm{CC}$ & 294 & 294 & 8 \\
\hline Peptidase M, neutral zinc metallopeptidase, zinc binding & INTERPRO & 5 & 8 & 4 \\
\hline Zinc finger, $\mathrm{C} 2 \mathrm{H} 2$-type & INTERPRO & 10 & 10 & 4 \\
\hline Ligase activity, forming carbon-nitrogen bonds & MF & 13 & 19 & 6 \\
\hline Cytosolic large ribosomal subunit & $\mathrm{CC}$ & 6 & 11 & 5 \\
\hline
\end{tabular}

Cluster 4: 254 transcripts

RNA metabolic process

Cellular metabolic process

Monooxygenase activity

\section{BP}

BP

MF

\section{3}

108

5
85

117

9
14

4

7

Cluster 5: 358 transcripts

Inflammatory response

Adaptive immune response

\section{BP}

$\mathrm{BP}$
21

8
12 
Table 1: Over-represented ontologies of each gene cluster (Continued)

\begin{tabular}{|c|c|c|c|c|}
\hline Pancreatitis-associated protein & INTERPRO & 4 & 7 & 6 \\
\hline Chemokine activity & MF & 5 & 10 & 10 \\
\hline Ras & INTERPRO & 10 & 20 & 8 \\
\hline GTP binding & MF & 19 & 19 & 8 \\
\hline Biopolymer modification & $\mathrm{BP}$ & 5 & 6 & 5 \\
\hline Immunoglobulin subtype & INTERPRO & 9 & 12 & 8 \\
\hline Cytokine production & $\mathrm{BP}$ & 6 & 10 & 8 \\
\hline \multicolumn{5}{|l|}{ Cluster 6: 186 transcripts } \\
\hline Glycosyltransferase & PIR & 8 & 10 & 12 \\
\hline Ribonucleotide binding & MF & 32 & 37 & 8 \\
\hline Contractile fiber part & $\mathrm{CC}$ & 4 & 4 & 4 \\
\hline Ras & INTERPRO & 6 & 10 & 8 \\
\hline Tyrosine-specific protein kinase & PIR & 4 & 10 & 9 \\
\hline Kinase activity & MF & 18 & 25 & 19 \\
\hline Hydrolase activity, hydrolysing O-glycosyl compounds & MF & 5 & 9 & 4 \\
\hline Biopolymer modification & $\mathrm{BP}$ & 28 & 60 & 6 \\
\hline
\end{tabular}

Cluster 7: 178 transcripts

Positive regulation of transcription from RNA polymerase II

Cluster 8: 238 transcripts

\begin{tabular}{llrrr}
\hline EGF-like & INTERPRO & 7 & 7 & 10 \\
Anatomical structure development & BP & 43 & 66 & 75 \\
Membrane part & CC & 75 & 4 \\
Transporter activity & MF & 32 & 35 \\
Transcription coactivator activity & MF & 8 & 14 & 8 \\
Intracellular transport & BP & 23 & 26 & 8 \\
Integral to endoplasmic reticulum membrane & CC & 5 & 7 \\
Coated vesicle & CC & 9 & 11
\end{tabular}

Cluster 9: 335 transcripts

\begin{tabular}{|c|c|c|c|c|}
\hline Protein amino acid phosphorylation & $\mathrm{BP}$ & 28 & 64 & 14 \\
\hline Plasma membrane part & $\mathrm{CC}$ & 31 & 53 & 4 \\
\hline Protein kinase, core & INTERPRO & 16 & 22 & 6 \\
\hline Immunoglobulin-like & INTERPRO & 13 & 13 & 5 \\
\hline Neurotransmitter transporter activity & MF & 5 & 12 & 14 \\
\hline System development & BP & 52 & 72 & 5 \\
\hline Gated channel activity & MF & 13 & 28 & 28 \\
\hline Neurological system process & $\mathrm{BP}$ & 29 & 30 & 4 \\
\hline ATP binding & MF & 37 & 45 & 8 \\
\hline Cation transmembrane transporter activity & MF & 19 & 39 & 8 \\
\hline Negative regulation of fibroblast proliferation & $\mathrm{BP}$ & 4 & 10 & 11 \\
\hline
\end{tabular}


Table 1: Over-represented ontologies of each gene cluster (Continued)

\begin{tabular}{|c|c|c|c|c|}
\hline Axogenesis & $\mathrm{BP}$ & 9 & 18 & 14 \\
\hline Regulation of neurotransmitter levels & $\mathrm{BP}$ & 9 & 14 & 6 \\
\hline \multicolumn{5}{|l|}{ Cluster 10: 263 transcripts } \\
\hline Membrane part & $\mathrm{CC}$ & 98 & 111 & 4 \\
\hline Glycolysis & $\mathrm{BP}$ & 13 & 37 & 25 \\
\hline Transporter activity & MF & 40 & 40 & 6 \\
\hline Purine ribonucleotide binding & MF & 45 & 48 & 9 \\
\hline Positive regulation of nucleobase, nucleoside, nucleotide & $\mathrm{BP}$ & 13 & 15 & 5 \\
\hline Synaptic transmission & $\mathrm{BP}$ & 17 & 31 & 5 \\
\hline Phosphorylation activity & $\mathrm{BP}$ & 24 & 49 & 25 \\
\hline Active transmembrane transporter activity & MF & 16 & 31 & 47 \\
\hline Phosphorylation activity & MF & 22 & 35 & 6 \\
\hline Neuron differentation & $\mathrm{BP}$ & 14 & 20 & 14 \\
\hline Cell development & $\mathrm{BP}$ & 34 & 37 & 11 \\
\hline Monovalent inorganic cation homeostasis & $\mathrm{BP}$ & 5 & 12 & 10 \\
\hline Amine transport & $\mathrm{BP}$ & 7 & 12 & 4 \\
\hline Ion exchanger activity & $\mathrm{BP}$ & 10 & 10 & 11 \\
\hline
\end{tabular}

Cluster 11: 251 transcripts

\begin{tabular}{llrrr}
\hline Calycin & INTERPRO & 10 & 10 & 6 \\
Synaptic transmission & BP & 18 & 27 & 5 \\
DNA repair & BP & 10 & 21 \\
Neuron projection development & BP & 11 & 24 \\
Developmental process & BP & 61 & 61 \\
Glycealdehyde-3-phosphate dehydrogenase () & MF & 5 & 20 \\
Activation of adenylatecyclase activity by G-protein signaling & BP & 4 & 9 \\
pathway & & & 21
\end{tabular}

Cluster 12: 333 transcripts

\begin{tabular}{llcc}
\hline Mitochondrial ATP synthesis coupled electron transport & BP & 10 & 19 \\
Mitochondrial part & CC & 31 & 44 \\
Oxidative phosphorylation & KEGG & 21 & 28 \\
Primary active transmembrane transporter activity & MF & 15 & 26 \\
Tricarboxylic acid cycle & BP & 6 & 9 \\
lon transmembrane transporter activity & MF & 23 & 37 \\
ATPase activity, coupled to transmembrane movement of & MF & 12 & 23 \\
substances & & 8 & 39 \\
Alkali metal ion binding (K+) & MF & 11 \\
Anion channel activity & MF & 5 & 14 \\
\hline
\end{tabular}

Class Ontology: $\mathrm{MF}=$ molecular function, $\mathrm{BP}=$ biological process, $\mathrm{CC}=$ cellular component, $\mathrm{PIR}=$ protein information resource. Count: Number of genes in ontology. Total: Number of genes in ontology cluster. Terms: number of ontology terms in ontology cluster. 
responses. Profiles 6 and 7, though being similar in their expression pattern, have little overlap in their over-represented ontologies. Profile 6 seems to reflect activation of a broad repertoire of kinase signaling pathways as well as modification of the ribosomal machinery, which might correlate with the only over-represented term in profile 7 , indicating a positive regulation of the transcriptional machinery relating to polymerase II. Profiles 8-11 all seem to contain ontologies relating to membrane bound activities such as active transport and neuronal signaling as well ontologies pertaining to engagement into neuronal developmental processes.

In conclusion, the ontology analysis ascribes several general functions to each time profile identifying their timing across the injury response and suggesting a common regulatory control of these.

\section{Differential expression of genes affecting motor neuron excitability}

While the ontology analysis may identify general terms subject to regulation, a manifest focus of our study was to correlate changes in gene expression that can be linked to increased motor neuron excitability and injury-induced spasticity. In a previous study we examined the late (21 and 60 days post-injury) transcriptional response of motor neurons to their sham-operated counterpart [32]. That study focused on changes in three main categories: ion channels, receptors of neurotransmitters and intracellular pathways capable of modulating these. Here we extract the same categories of significantly differentially expressed genes. These genes are shown in Table 2 along with the cluster identity. The majority of these differentially expressed genes are identical to the genes reported in our previous study [32], with the two primary differences being that in the present case they are, 1) identified based on their differential expression across time as opposed to a static comparison to their sham-operated counterpart at each time point and 2) each gene is associated with a pattern of expression over time. Most importantly, we thus identify the same gene candidates subject to regulation with two different strategies for the choice of reference, supporting the robustness of our findings. But, in contrast to the static study (25), the dynamic response over time now enables us to expand our analysis and start unraveling some of the underlying regulatory mechanism shaping the observed patterns of gene expression within each cluster profile.

To summarize the regulation of genes directly relating to motor neuron excitability, we find that most of the neuromodulator pathways (serotonergic, dopaminergic and adrenergic) seem to have a response of late up-regulation (clusters 8-10) while inhibitory neurotransmitter pathways (GABAergic and glycinergic) are in general down-regulated in the late phase of the injury response
Table 2: Differentially expressed genes relating to motor neuron excitability

\begin{tabular}{lllr}
\hline Probe IDs & Gene ID & Protein ID & Cluster \\
\hline Calcium Channels & & & \\
\hline 1371039 at & Cacnb4 & CAB4 & 8 \\
\hline 1368398 at & Cacna1h & Cav3.2 & 9 \\
\hline 1371175 a_at & Cacna1b & Cav2.2 & 10 \\
\hline 1369706 at & Cacng1 & Cacng1 & 11 \\
\hline 1386939 a_at & Cacna1a & Cav2.1 & \\
\hline
\end{tabular}

Sodim Channels

\begin{tabular}{lllr}
\hline 1379307_at & Sap1 & SAP1 & 1 \\
\hline 1369662_at & Scn2a1 & Nav1.2 & 3 \\
\hline 1368539_at & Scn9a & Nav1.7 & 5 \\
\hline 1383435_at & Scn3b & SCN3B & 5 \\
\hline 1370850_at & Scn3b & SCN3B & S \\
\hline 1387010_s_at & Scn1b & SCN1B & 8 \\
\hline 1368351_at & Scn10a & Nav1.8 & 9 \\
\hline 1388035_a_at & Scn5a & Nav1.5 &
\end{tabular}

\section{Potassium Channels}

\begin{tabular}{lllr}
\hline 1370439_a_at & Kcnc2 & Kv3.2 & 1 \\
\hline 1369043_at & Kcna4 & Kv1.4 & 2 \\
\hline 1386770_x_at & Kcne2 & KCNE2 & 2 \\
\hline 1385226_at & Kctd11 & KCD11 & 3 \\
\hline 1387264_at & Kcnk6 & TASK-1 & 5 \\
\hline 1370958_at & Kcnc3 & Kv3.3 & 8 \\
\hline 1389120_at & Kcnc3 & Kv3.3 & 9
\end{tabular}


Table 2: Differentially expressed genes relating to motor neuron excitability (Continued)

\begin{tabular}{|c|c|c|c|}
\hline 1369847_at & Kcnab1 & KCAB1 & 9 \\
\hline 1369280_at & Kcnk9 & TASK-3 & 9 \\
\hline 1370595_a_at & Kcnip4 & KCIP4 & 9 \\
\hline 1370558_a_at & Kcnc2 & $\mathrm{Kv} 3.2$ & 9 \\
\hline 1387477_at & Kcnk12 & THIK-2 & 10 \\
\hline 1370545_at & Kena1 & Kv1.1 & 11 \\
\hline 1368343_at & Kenh2 & Kv11.2 & 11 \\
\hline 1368751_at & Kcns3 & Kv9.3 & 12 \\
\hline 1374582_at & Kctd9 & KCD9 & 12 \\
\hline 1368524_at & Kenc1 & Kv3.1 & 12 \\
\hline 1370076_at & Kcnj16 & Kir5.1 & 12 \\
\hline
\end{tabular}

\begin{tabular}{lllr}
\hline Chloride Channels & & & \\
\hline 1367772_at & Clns1a & ICLN & 1 \\
\hline 1367893_a_at & Clcc1 & CLCC1 & \\
\hline 1378658_at & Clca6 & CLCA6 & \\
\hline 1392453_at & Clcn3 & CLCN3 & 10 \\
\hline 1380547_at & Clcn3 & CLCN3 & 10 \\
\hline 1379932_at & Clcn4-2 & CLCN4-2 & 12
\end{tabular}

\section{Calmodulin and CaM kinase}

\begin{tabular}{lllr}
\hline 1369993_at & Camk2g & KCC2G & 9 \\
\hline 1398251_a_at & Camk2b & KCC2B & 9 \\
\hline 1369937_at & Calm1 & CALM & 11 \\
\hline 1370853_at & Camk2n1 & CK2N1 & 11 \\
\hline 1368101_at & Calm3 & CALM & 12
\end{tabular}

Table 2: Differentially expressed genes relating to motor neuron excitability (Continued)

\begin{tabular}{|c|c|c|c|}
\hline \multicolumn{4}{|c|}{ Calcium binding proteins } \\
\hline 1369886_a_at & Cabp1 & $\mathrm{CaBP} 1$ & 11 \\
\hline \multicolumn{4}{|l|}{ IP3 } \\
\hline 1368005_at & It $r 3$ & ITPR3 & 7 \\
\hline \multicolumn{4}{|c|}{ Glutamate Receptors } \\
\hline 1387286_at & Grm1 & mGluR1 & 1 \\
\hline 1398889_at & Grinl1a & GL1AD & 1 \\
\hline 1396696_at & Gria4 & GluR4 & 3 \\
\hline 1369036_at & Grik2 & GRIK2 & 4 \\
\hline 1368572_a_at & Grin1 & NR1 & 8 \\
\hline 1368759_at & Cacng2 & CCG2/TARP & 10 \\
\hline 1372724_at & Grina & NMDARA1 & 10 \\
\hline 1369128_at & Grik5 & GRIK5 & 10 \\
\hline 1387559_at & Grin3b & NMDA3B & 11 \\
\hline \multicolumn{4}{|c|}{ GABA Receptors } \\
\hline 1368170_at & Slc6a1 & GAT1 & 1 \\
\hline 1380170_at & Gabarap/2 & GBRL2 & 1 \\
\hline 1380828_at & Gabra1 & GBRA1 & 1 \\
\hline 1391653_at & Gabrg2 & GBRG2 & 1 \\
\hline 1370702_at & Gabrr3 & GBRR3 & 9 \\
\hline 1370804_at & Gabarap & GBRAP & 10 \\
\hline 1378842_at & Gabarapl1 & GBRL1 & 11 \\
\hline 1387383_at & Gabbr2 & GBRR2 & 12 \\
\hline 1369904_at & Gabrb1 & GBRB1 & 12 \\
\hline
\end{tabular}


Table 2: Differentially expressed genes relating to motor neuron excitability (Continued)

\begin{tabular}{|c|c|c|c|}
\hline 1367783_at & Gabarap/2 & GBRL2 & 12 \\
\hline \multicolumn{4}{|c|}{ Glycine Receptors } \\
\hline 1387696_a_at & Glra2 & GLRA2 & 2 \\
\hline \multicolumn{4}{|c|}{ Cholinergic Receptors } \\
\hline 1370607_a_at & $\mathrm{Nrg} 1$ & NRG1 & 9 \\
\hline 1369845_at & Chrna6 & ACHA6 & 9 \\
\hline 1369252_a_at & Chrna4 & $\mathrm{ACHA} 4$ & 9 \\
\hline 1368615_a_at & Slc18a3 & VAChT & 10 \\
\hline 1368734_at & Chrnd & $\mathrm{ACHD}$ & 10 \\
\hline \multicolumn{4}{|c|}{ Serotonin Recoptors } \\
\hline 1369456_at & $H \operatorname{tr} 2 b$ & $5 \mathrm{HT} 2 \mathrm{BR}$ & 5 \\
\hline 1369119_a_at & Htr7 & 5HT7BR & 11 \\
\hline \multicolumn{4}{|c|}{ Adrenergic Receptors } \\
\hline 1368534_at & Adrald & ADA1D & 8 \\
\hline 1388757_at & Adrbk1 & ARBK1 & 10 \\
\hline 1369797_at & Adrala & ADA1A & 11 \\
\hline
\end{tabular}

\section{Dopamin Receptors}

\begin{tabular}{lllr}
\hline 1368602_at & Slc6a3 & DAT & 6 \\
\hline 1368601_at & Slc6a3 & DAT & 9 \\
\hline $1387520 \_a t$ & Drd4 & DRD4 & 9 \\
\hline 1369856_at & Drd5 & DRD5 & 11 \\
\hline 1376345_at & Drd1ip & (Caly) CALY & 12
\end{tabular}

Cannabinoid Receptor
Table 2: Differentially expressed genes relating to motor neuron excitability (Continued)

\begin{tabular}{lllr}
\hline Anion Transporters & & & \\
\hline 1367853 at & Slc12a2 & NKCC1 & 1 \\
\hline $1368082 \_a t$ & Slc4a2 & AE2 & 10 \\
\hline 1368772 at & Slc4a3 & AE3 & 10 \\
\hline
\end{tabular}

(clusters 1-2 and 12). The time series analysis also reveals regulation of genes coding for serotonergic (Htr7) and adrenergic (Adrala) receptors, which were not seen in the analysis of the late injury response [32] because they belong to time profile 11 with an initial suppression followed by a return to control levels. Other adrenergic receptor related genes overlap in the two studies, alpha 1D adrenoreceptor (Adrald, profile 8 ) and beta-adrenergic receptor kinase 1 (Adrbkl, profile 10) being up-regulated in the late phase. The dopamine reuptake transporter DAT (profiles 6 and 9) together with the gene coding for the dopamine receptor 4 ( $D r d 4$, profile 9) are up-regulated while a gene coding for the dopamine interacting protein Caly (profile 12) is down-regulated. The gene coding for dopamine receptor 5 ( $D r d 5$, profile 11) is subject to early down-regulation 2 and 7 days post injury, but returns to control levels in the late phase 60 days post injury. As in the preceding study [32] we find genes coding for GABAA subunits involved in channel trafficking and membrane incorporation to respond to the injury (Gabarap, profile10; Gaparapll, profile 11; Gaparapl2, profiles 1 and 12) in synergy with the down-regulation of the receptor subunits $\mathrm{GABA}_{\mathrm{A}} \alpha_{1}$ (Gabral, profile 1), $\mathrm{GABA}_{\mathrm{A}} \gamma_{2}$ (Gabrg2, profile 1) and $\mathrm{GABA}_{\mathrm{A}}$ receptor $\beta_{1}$ (Gabrbl, profile 12). One additional gene relating to GABA transmission is down-regulated, the $\mathrm{GABA}_{\mathrm{B}}$ receptor 2 (Gabbr2, profile 12).

The time analysis showed in accordance with [32] that the glutamatergic receptors seem to undergo a complex regulation, where several genes coding for different components of the NMDA receptor undergo regulation in late stages: Grinl (profile 8) and Grina (profile 10) are upregulated and Grinlla (profile 1) is down-regulated. Grin3b also belong to the NMDA receptor complex, but seems to undergo early modulation with early down-regulation and a return to control levels in late phases (profile 11). The gene coding for the AMPA receptor regulator protein TARP is up-regulated (Cacng2 (stargazine), profile 10), suggesting an increased AMPA receptor mediated conductance.

With respect to the cholinergic system the time series analysis revealed similar patterns as was seen in the anal- 
ysis of the late injury response [32], all genes being upregulated in the late phases of the response. The up-regulation of genes coding for nicotinic alpha receptors 4 and 6 (Chrna4 and Chrana6, profile 9) together with the receptor subunit delta (Chrnd, profile 10) suggest an increased sensitivity to acetylcholine, while the up-regulation of the genes coding for vesicular acetylcholine transporter (VAChT, profile 10) as well as for NRG1 (Nrg1, profile 10) know to be involved in synaptic maturation suggest and increased release of acetylcholine.

Among the voltage gated ion channels, genes coding for $\mathrm{Ca}^{2+}$ and $\mathrm{Na}^{+}$channel subunits are largely up-regulated in the late phase, while genes relating to $\mathrm{K}^{+}$have a more complex response with a balanced up- and down regulation of channel subunits. The genes coding for the $\mathrm{Ca}^{2+}$ channel $\alpha$ subunits Cav3.2, Cav2.2 and the $\gamma_{1}$ subunit (Cacng1, profile 10; Caclh, profile 8; Cacna1b, profile 9) are up-regulated in the late phase of the injury response, while only the gene coding for the $\mathrm{Ca}^{2+}$ channel subunit $\beta_{4}$ (Cacnb4, profile 4) exhibit a late down-regulation. Several genes of the $\mathrm{Na}^{+}$subunits also undergo regulation, where the genes coding for $\mathrm{Na}^{+}$a subunits Nav1.8 and Nav1.5 together with the $\beta_{1}$ subunit (Scn10a, profile 8; $S c n 5 a$, profile 9; $S c n 1 b$, profile 8) exhibit late up-regulation, while only the gene coding for the a subunit Nav1.2 (Scn2a1) is down-regulated. Both the genes coding for the a subunit Nav1.7 (Scn9a) and the $\beta_{3}$ subunit (Scnb3) belong to profile 5 with an early up-regulation and a return to control levels in the late phases. $\mathrm{Ca}^{2+}$ binding proteins also exhibit a trend towards late phase up-regulation, i.e. CaM kinase related genes (Camk2g and Camk $2 b$, profile 9) as well as one $\mathrm{IP}_{3}$ receptor (Itpr3, profile 7) and the $\mathrm{Ca}^{2+}$ binding protein caldendrin (Cabp1, profile 11) are up-regulated. Two genes relating to calmodulin and CaM kinase (Calm1 and Camk2n1, profile 11) are transiently down-regulated in the early injury response, returning to control levels in the late part of the injury-response. The $\mathrm{Cl}^{-}$reversal potential also seems to be subject to regulation towards a more depolarizing effect, suggested by the down-regulation of the gene coding for the $\mathrm{Cl}^{-}$transporter NKCC1 (Slc12a2, profile 1) responsible for $\mathrm{Cl}^{-}$extrusion and the up-regulation of the gene coding for the $\mathrm{Cl}^{-}$symporters AE2 and AE3 (Slc4a2 and $\mathrm{Slc4a3}$, profile 10) involved in $\mathrm{Cl}$-accumulation inside the cell.

\section{The transcriptional regulation exerted by differentially expressed transcription factors}

The common expression patterns of each consensus cluster suggest a common regulatory control of their associated genes. To reveal such common regulatory control, we looked for over-representation of transcription factor DNA binding sites in their proximal promoter regions, here set to 1000 base pairs (bp) upstream and $200 \mathrm{bp}$ downstream of the coding region.

We focused the analysis on transcription factors that were associated with genes that exhibit differential expression in the time series. We find 34 genes coding for transcription factors to be differentially expressed in our data set out of the 119 transcription factor genes contained on the RAT230 2 chip. These genes are listed in Table 3 along with their cluster identity. To evaluate if these factors are likely to exert an influence on the regulation of the genes in each cluster, we predict their binding sites in respective gene promoters using computational models (see [37] for a review). Over-represented binding sites suggest a possible role for the corresponding transcription factor in the regulation of the tested set of genes. For this analysis we use ASAP [38] with position weight matrices (PWM) obtained from either JASPAR [39] or TRANSFAC [40] and the set of differentially expressed gene promoter sequences as background.

There is presently little overlap between the transcription factors associated with the genes included on the RAT230 2 chip and the two databases containing their binding motifs, only 29 overlap with TRANSFAC and 10 with JASPAR (Figure 2A-B). Of these transcription factors (with both expression data and motif annotation), the ones associated with genes that do not display differential expression were excluded from the analysis, reducing the two sets of transcription factors to six for TRANSFAC (ATF3, ATF4, MYC, FOXO1, SP1 and E2F1) and two for JASPAR (SP1 and E2F1). We therefore used the TRANSFAC motifs for the promoter analysis. Because many of the TRANFAC motifs describe the same factor, this analysis includes 16 motifs. Patterns with a substantial enrichments are reported $(Z$ score $>3$, as in [38]), since these are likely to exert an influence in the regulation of the gene clusters, Table 3 . Under-represented binding motifs signify that the corresponding transcription factor is very unlikely to exert any regulatory influence on the gene cluster under examination $(\mathrm{Z}$ score $<-3$, not included in this table).

To illustrate the balance between over- and under-representation of binding sites across gene clusters we next take advantage of the continuous range of $\mathrm{Z}$ scores, instead of only treating them as binary classifiers (overrepresentation or not). In combination across the gene clusters these values say something about the regulatory landscape, i.e. what binding sites are unchanged in most clusters, and what factors can explain the difference between clusters? We choose to visualize this as a hierarchical heatmap, where rows constitute the motif models and columns the gene clusters. In this representation the $\mathrm{Z}$ scores are organized by two-way hierarchical clustering, such that motifs that behave similarly in terms of over-representation will cluster together, as will the gene 
Table 3: Differentially expressed genes of transcription factors (TF) and over-representation (over-rep) of TF binding sequences within each gene cluster

\begin{tabular}{|c|c|c|c|c|}
\hline \multirow[b]{2}{*}{ Clusters } & \multicolumn{2}{|c|}{ DEG of TF } & \multirow[b]{2}{*}{ TF over-rep } & \multirow[b]{2}{*}{ Motifs } \\
\hline & Gene ID & Protein ID & & \\
\hline \multirow[t]{5}{*}{ Cluster 1} & Gtf2e2 & T2EB & $\mathrm{E} 2 \mathrm{~F} 1$ & E2F1_Q3_01 \\
\hline & Nfyc & NFYC & FOXO1 & E2F1_Q2_01 \\
\hline & Olig1 & OLIG1 & & E2F1_Q6_01 \\
\hline & $P b \times 3$ & PBX3 & & FOX01_01 \\
\hline & $T f b 2 m$ & TFB2M & & FOXO1_02 \\
\hline \multirow[t]{2}{*}{ Cluster 2} & $E 2 f 5$ & E2F5 & FOXO1 & FOX01_01 \\
\hline & Morf4l1 & MO4L1 & & FOXO1_02 \\
\hline \multirow[t]{3}{*}{ Cluster 3} & Aatf & AATF & FOXO1 & FOX01_01 \\
\hline & Btf3 & BTF3 & & FOXO1_02 \\
\hline & KIf10 & KLF10 & & \\
\hline \multirow[t]{3}{*}{ Cluster 4} & Arid1b & ARID1B & $\mathrm{E} 2 \mathrm{~F} 1$ & E2F1_Q3_01 \\
\hline & Gtf2ird1 & GT2D1 & FOXO1 & FOXO1_01 \\
\hline & $\operatorname{Irf9}$ & IRF9 & & FOXO1_02 \\
\hline \multirow[t]{4}{*}{ Cluster 5} & Atf3 & ATF3 & ATF4 & ATF4_Q2 \\
\hline & Myc & MYC & & \\
\hline & Tceb3 & ELOA1 & & \\
\hline & $Y b \times 1$ & YBX1 & & \\
\hline \multirow[t]{8}{*}{ Cluster 6} & & & ATF4 & ATF4_Q2 \\
\hline & & & $\mathrm{E} 2 \mathrm{~F} 1$ & E2F1_Q3 \\
\hline & & & MYC & MYC_Q2 \\
\hline & & & SP1 & SP1_01 \\
\hline & & & & SP1_Q2_01 \\
\hline & & & & SP1_Q4_01 \\
\hline & & & & SP1_Q6 \\
\hline & & & & SP1_Q6_01 \\
\hline \multirow[t]{12}{*}{ Cluster 7} & $E 2 f 1$ & E2F1 & ATF3 & ATF3_Q6 \\
\hline & Foxo1 & F0X01 & ATF4 & ATF4_Q2 \\
\hline & Gtf3c1 & TF3C1 & E2F1 & E2F1_Q3 \\
\hline & Sp1 & SP1 & MYC & E2F1_Q3_01 \\
\hline & & & SP1 & E2F1_Q4 \\
\hline & & & & E2F1_Q4_01 \\
\hline & & & & E2F1_Q6 \\
\hline & & & & E2F1_Q6_01 \\
\hline & & & & MYC_Q2 \\
\hline & & & & SP1_01 \\
\hline & & & & SP1_Q2_01 \\
\hline & & & & SP1_Q4_01 \\
\hline
\end{tabular}


Table 3: Differentially expressed genes of transcription factors (TF) and over-representation (over-rep) of TF binding sequences within each gene cluster (Continued)

\begin{tabular}{|c|c|c|c|c|}
\hline & & & & SP1_Q6 \\
\hline & & & & SP1_Q6_01 \\
\hline \multirow[t]{11}{*}{ Cluster 8} & $H s f 4$ & HSF4 & ATF3 & ATF3_Q6 \\
\hline & $T b \times 3$ & $T B X 3$ & ATF4 & ATF4_Q2 \\
\hline & & & $\mathrm{E} 2 \mathrm{~F} 1$ & E2F1_Q3 \\
\hline & & & MYC & E2F1_Q3_01 \\
\hline & & & SP1 & E2F1_Q6 \\
\hline & & & & MYC_Q2 \\
\hline & & & & SP1_01 \\
\hline & & & & SP1_Q2_01 \\
\hline & & & & SP1_Q4_01 \\
\hline & & & & SP1_Q6 \\
\hline & & & & SP1_Q6_01 \\
\hline \multirow[t]{8}{*}{ Cluster 9} & Arid $1 b$ & $A R I D 1 B$ & ATF3 & ATF3_Q6 \\
\hline & $N r 2 f 2$ & COT2 & $\mathrm{E} 2 \mathrm{~F} 1$ & E2F1_Q3 \\
\hline & Pou2f3 & $\mathrm{PO} 2 \mathrm{~F} 3$ & SP1 & E2F1_Q6 \\
\hline & Runx3 & RUNX3 & & SP1_01 \\
\hline & & & & SP1_Q2_01 \\
\hline & & & & SP1_Q4_01 \\
\hline & & & & SP1_Q6 \\
\hline & & & & SP1_Q6_01 \\
\hline \multirow[t]{13}{*}{ Cluster 10} & Atf4 & ATF4 & ATF4 & ATF4_Q2 \\
\hline & Srebf1 & SRBP1 & E2F1 & E2F1_Q3 \\
\hline & & & MYC & E2F1_Q3_01 \\
\hline & & & SP1 & E2F1_Q4 \\
\hline & & & & E2F1_Q4_01 \\
\hline & & & & E2F1_Q6 \\
\hline & & & & E2F1_Q6_01 \\
\hline & & & & MYC_Q2 \\
\hline & & & & SP1_01 \\
\hline & & & & SP1_Q2_01 \\
\hline & & & & SP1_Q4_01 \\
\hline & & & & SP1_Q6 \\
\hline & & & & SP1_Q6_01 \\
\hline \multirow[t]{8}{*}{ Cluster 11} & Dmrt1 & $D M R T 1$ & AFT3 & ATF3_Q6 \\
\hline & Gtf2h4 & GTF2H4 & $\mathrm{E} 2 \mathrm{~F} 1$ & E2F1_Q3 \\
\hline & Zeb1 & ZEB1 & MYC & MYC_Q2 \\
\hline & & & SP1 & SP1_01 \\
\hline & & & & SP1_Q2_01 \\
\hline & & & & SP1_Q4_01 \\
\hline & & & & SP1_Q6 \\
\hline & & & & SP1_Q6_01 \\
\hline
\end{tabular}


Table 3: Differentially expressed genes of transcription factors (TF) and over-representation (over-rep) of TF binding sequences within each gene cluster (Continued)

\begin{tabular}{|c|c|c|c|c|}
\hline \multirow[t]{6}{*}{ Cluster 12} & Nfia & NFIA & AFT3 & ATF3_Q6 \\
\hline & $N k \times 6-2$ & NKX6-2 & $\mathrm{E} 2 \mathrm{~F} 1$ & E2F1_Q3_01 \\
\hline & & & FOXO1 & E2F1_Q4_01 \\
\hline & & & & E2F1_Q6 \\
\hline & & & & E2F1_Q6_01 \\
\hline & & & & FOXO1 01 \\
\hline
\end{tabular}

DEG TF: Differentially expressed genes (DEG) of transcription factors (TF). Genes of TF with annotated TRANSFAC motifs are highlighted in boldface. TF over-rep: TFs with over-represented binding sites in the set of genes belonging to the specified gene cluster. Motifs: Individual TRANSFAC motifs with Z scores above 3 based on ASAP conducted on the set of genes belonging to the specified gene cluster.

clusters with similar bindings site landscapes, Figure 3A. For plotting purposes each $\mathrm{Z}$ score vector (column) was normalized to unit variance. The heatmap of $Z$ scores shows a clear pattern separating the expression profiles into groups sharing over-represented (red) as well as under-represented (green) sites. Motifs whose sites are over-represented in the group of clusters 1-4 are underrepresented in the other major group clusters 6-11, and vise versa. The patterns of transcription factor binding site over- and under-representation are not identical for each of the consensus clusters, perhaps alluding to some degree of specific regulation within each cluster. Cluster profiles 5 and 12 have slightly separate motif binding patterns, though resembling clusters 6-11 and 1-4, respectively. The first two principal components of the $\mathrm{Z}$ score vectors of each time profile also reflect this clear separation, again reproducing the relationships obtained from the consensus clustering (Figure 1C), where profiles 1-4 and 6-11 group together, while clusters 5 and 12 are somewhat separated from these two main groups of regulation, Figure 3B.

By comparing the expression pattern of the differentially expressed genes coding for transcription factors (which cluster they belong to) with the time profile of the clusters where their binding sites are over-represented (target cluster) some general picture emerges. The differentially expressed genes of SP1 (Sp1) and E2F1 (E2f1) belong to cluster 7 , which show an early up-regulation that is maintained throughout the injury response. The pattern of binding site over-representation suggests that SP1 may have an auto regulatory role, as its binding sites are over-represented in cluster profiles 6-11 and underrepresented in cluster profiles $1-4$ and 12 . It thus seems to enhance expression of genes in clusters 6-11. The pattern of over-representation for the binding site of E2F1 on the other hand suggest that this transcription factor has a very broad activation potential as it seems to target clusters 1, 4 and 6-12. The expression of the gene coding for

\section{A}

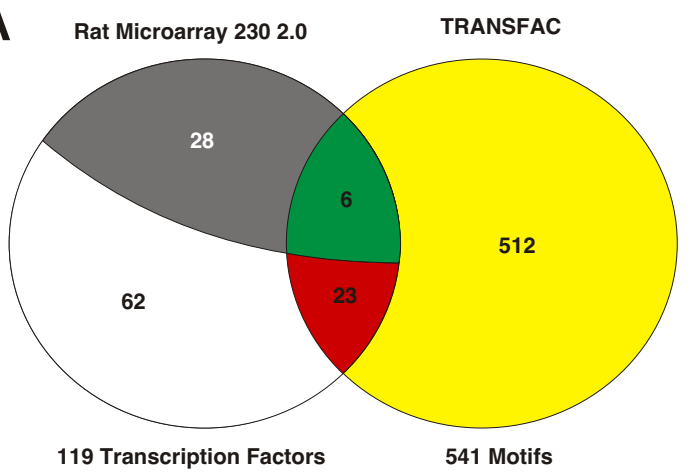

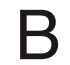

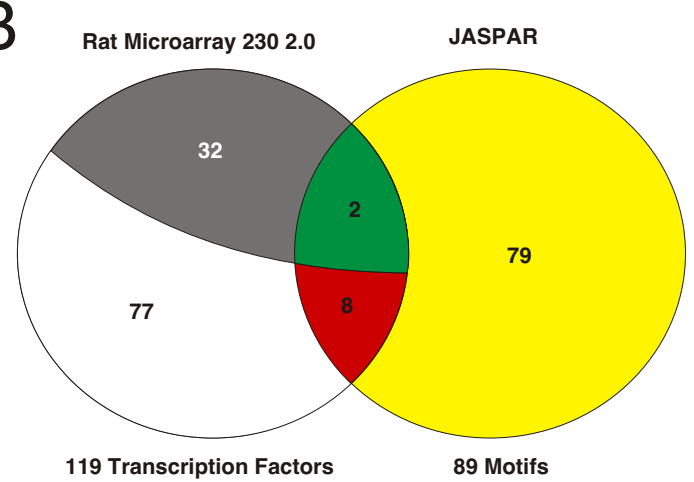

Figure 2 Overlap between transcription factors and their binding motifs. Transcription factor overlap between the 119 transcription factors included on the RAT230 2 chip and their binding motifs contained in TRANSFAC or JASPAR. A. Circle to the left represents the set of transcription factors included on the RAT230 2 chip, in total 119 of which 34 are differentially expressed. TRANFAC contains DNA binding motifs of 541 distinct transcription factors, 29 of which are on the array. Of these 6 are differentially expressed (green) and 23 are non-differentially expressed (red). Thus 28 (grey) differentially expressed transcription factors do not have binding motifs in TRANFAC, which on the other hand contains binding motifs of 512 transcription factors not contained on the microarray (yellow). B. Overlap between the transcription factors included on the RAT230 2 chip and their biding motifs contained in JASPAR. Same color code as in A. 
FOXO1 (Foxo1) follows time profile 7, and the over-represented binding sites of this transcription factor in clusters 1-4 and 12 suggests that it suppress the expression of genes in these clusters as they are mainly down-regulated. The gene of transcription factor ATF4 (Atf4) belongs to cluster profile 10 showing a late up-regulation. The binding sites of ATF4 are over-represented in cluster profile 5 so its late expression may be involved in the late suppression of genes in cluster profile 5 . Cluster profile 5 on the other hand contains two differentially expressed genes coding for transcription factors, MYC $(M y c)$ and ATF3 (Atf3). The binding sites of MYC are over-represented in the promoters of genes belonging to cluster profiles $6,7,8,10$ and 11 . This could indicate a role for MYC in the positive regulation in the early response (2 and 7 days post injury) of cluster profiles $6-8$, while it would have the opposite effect suppressing the expression of genes in clusters 10 and 11 (see Discussion). As the genes of cluster profile 5 reside to control levels in the late phase of the injury response (21 and 60 days post injury), it seems unlikely that transcription factors following this time profile exert any effect on gene regulation at these late time points. The binding sites of ATF3 are over-represented in profiles $7,8,9,11$ and 12 , suggesting that this transcription factor affects the early up-regulation in clusters 7 and 8 while the profile of cluster 9 doesn't seem to undergo any significant regulation at these time points compared to control (day 0). As for transcription factor MYC it seems likely that ATF3 participate in the suppression of gene expression in the early response in clusters 11 and 12.

This analysis showed that although we could only ascribe motifs to 6 of the 34 transcription factors encoded by differentially expressed genes, the complex correlation between the timing of their gene expression and the down- or up-regulation of their putative cluster targets suggests an intricate interaction between the transcription factors in shaping the transcriptional response.

\section{Discussion}

Excitability changes in motor neurons have been strongly implicated with the emergence of pathophysiological hyper-reflexia in late stages of spinal cord injury, since self-sustained activity can be induced in motor neurons upon brief stimuli of sensory afferents in the complete absence of descending fibers from the brain $[3,5,9,23]$. By focusing on the transcriptional time course of these cells in combination with transcription factor motif analysis we shed light on the regulatory mechanisms underlying the re-expression of these plateau potentials, a key mechanism behind the pathophysiology of spasticity. In particular, we use a robust consensus cluster algorithm [35] to identify distinct expression time profiles. This consensus cluster algorithm conducted on the 3,708 most differen- tially expressed genes identified 12 distinct time profiles. These expression time profiles separate the differentially expressed genes into groups that most likely are under common regulatory control and enable us to associate individual genes with a specific pattern of expression over time.

\section{Cluster analysis identify distinct time profiles that define the timing of general biological responses to injury}

The 12 time profiles divide into two main groups relating to the late response, one of down-regulation (time profiles 1-4 and 12) and one of up-regulation (time profiles 6-10), Figure $1 \mathrm{~B}$ and $1 \mathrm{C}$. Besides these two main categories of late regulation expression patterns there are two clusters with a predominant early response, time profile 5 with an early up-regulation at day 2 and time profile 11 with an early down-regulation at days 2 and 7 , which both falls back towards control levels 21 and 60 days post injury.

Ontology analysis of the genes associated with each cluster profile shows that the motor neurons engage in different biological processes as the transcriptional response evolves over time. In particular time profile 5 signifies a marked immunological and inflammatory response of the motor neurons in the early phase after injury, which return to control levels in the late phases. Such immunological processes are known to be pronounced in the early phase of spinal cord injury from studies conducted on entire spinal cord tissue [1,41-43], but have not previously been identified at the motor neuron level. This finding corroborate recent studies, indicating that a neuronal immune response is included in the repertoire of processes motor neurons can engage as a means of protection against damage [44]. Cluster profiles 1-4 as well as 12 all describe different patterns of transcript down-regulation. "Cell-cell adhesion" is clearly down-regulated in profiles 1 and 3, suggesting that the direct interaction of motor neurons with their neighboring cells are reduced. Synapse stripping, including the removal of synapses from the perikaryon and dendrites, is a pronounced phenomenon after axonal damage to motor neurons [44]. The down-regulation of genes related to "ensheathment of neurons" in cluster profile 1 suggests an effect of the injury on the myelination of motor neurons not previously associated with this neuronal population. Another prominent down-regulated mechanism involves mitochondrial related energy metabolism (time profile 12). The translational machinery is also down-regulated (profiles 2-3). The down-regulation of chromatin structures (profile 1) suggests that the DNA could be unfolding towards a more favorable transcriptional state, while "RNA splicing" of profile 2 suggests a reduction in the mRNA processing. Among the prominent up-regulated profiles, pathways relating to neuronal 

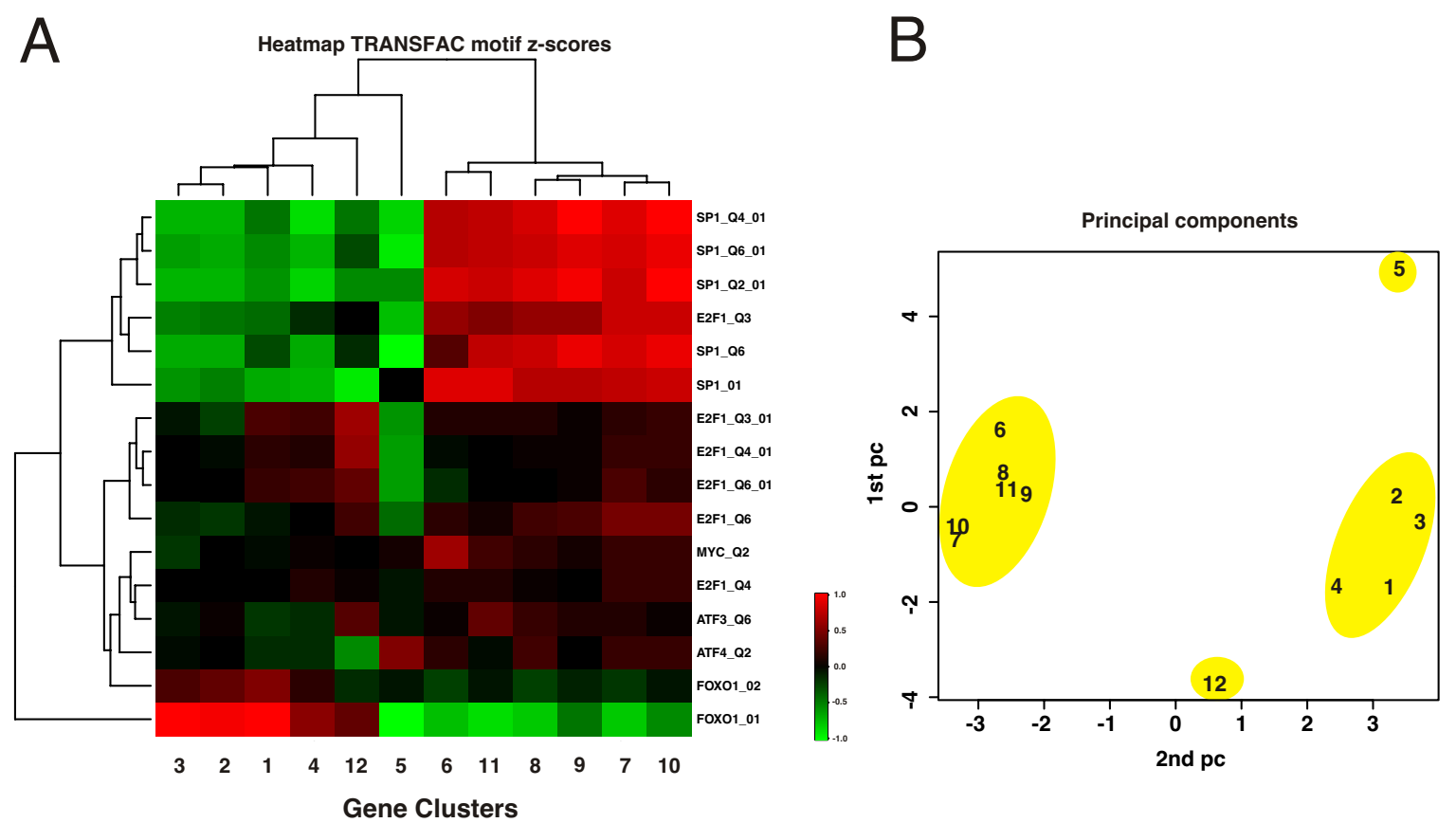

Figure 3 Motif over-representation of differentially expressed transcription factors. Over-representation statistics for each cluster profile of the 16 binding motifs contained in TRANSFAC representing the 6 differentially expressed transcription factors: ATF3, ATF4, E2F1, FOXO1, MYC, SP1. A. Heatmap of Z scores organized by two-way hierarchical clustering according to the Z score pattern across binding motifs and cluster profiles. For plotting purposes each Z score vector was normalized to unit variance. Over-represented genes are highlighted in red while under-represented transcription factors are shown in green (see color bar). It is clear that consensus clusters 1-4 and 6-11 group together, sharing common over- as well as underrepresented transcription factor motifs. It is also striking that motifs over-represented in these groups typically will be under-represented in the other and vice versa. Consensus clusters 5 and 12 have different patterns of binding motifs Z scores. B. Principal components of the Z score vectors of each consensus cluster (columns of A) validate the pattern observed in A: cluster profiles 1-4 and 6-11 are closely grouped while cluster profiles 5 and 12 are separated from these.

development (profiles 8-10), suggest that injury induce developmental processes as a late response. This finding indicates that a differentiated and mature neural population in the spinal cord is capable of re-engaging in developmental pathways, presumably attempting to ameliorate the conditions of the damaged spinal cord and compensate for the lack of inputs. It is also clear that plasma membrane transporter activity of various kinds are significantly up-regulated suggesting a very strong control of the electro-chemical transmembrane gradients, possibly also reflecting the changing chemical requirements of the motor neurons. The motor neurons also up-regulate processes directly relating to membrane excitability and neural transmission, suggesting that the motor neurons change their synaptic strength, both pre-synaptically through modulation of axon terminals with increased machinery for acetylcholine release and post-synaptically through modulation of receptor channels as well as changed membrane excitability.

\section{Differentially expressed genes relating to motor neuron excitability and injury-induced spasticity}

Ontology analysis provides general terms of activity suggesting some biological functions of each cluster profile, but the over-represented ontologies only represent a relative small proportion of the genes contained in each cluster. To dissect out all the gene constituents that relate to changes in motor neuron excitability and injury-induced spasticity we therefore focused on genes involved in neural transmission. In a previous study we examined the late transcriptional response of motor neurons compared to their sham-operated counterparts 21 and 60 days post injury [32]. From this study it was clear that the motor neurons change their post-synaptic receptor composition moving towards a more excitable state through a reduction of the ionotropic GABAergic receptors and an increase of the ionotropic glutamatergic, adrenergic and cholinergic receptors. $\mathrm{Ca}^{2+}$ and $\mathrm{Na}^{+}$ion channels also responded to the injury, where the most noticeable changes related to the modulation of persistent inward currents involved the ancillary subunits possibly changing the conductivity and membrane incorporation of existing ion channels. The functional consequences of these changes are discussed extensively in [32].

Extracting the differentially expressed genes affecting motor neuron excitability based on their changed expression over time identifies many of the same candidates, 
though the differential expression in the present case is based on mutual reference across the time points rather than pair wise comparisons within each time point with time-matched sham controls. The fact that the two different strategies of analysis identify many of the same gene candidates affecting motor neuron excitability supports the robustness of our findings. The time series analysis clearly shows that most of the genes relating to motor neuron excitability and injury-induced spasticity are found in clusters with late regulation, where genes coding for receptors and channels with excitatory effects are predominantly up-regulated while inhibitory receptors are primarily down-regulated. This shows that the observed progressive increase of the hyper-reflexia strongly correlates with the increased expression of genes enhancing motor neuron excitability and reversely correlates with the decreasing expression of the $\mathrm{GABA}_{\mathrm{A}}$ receptor system. This pattern of expression was also reflected in the ontology analysis, where late phase up-regulation of time profiles 9 and 10 contains terms of "gated channel activity", "regulation of neurotransmitter levels", "synaptic transmission" and "ion exchanger activity", while time profile 12 showing a general down-regulation as a response to the injury contains "alkali metal ion binding" and "anion channel activity" reflecting a decrease in anion channel signaling $\left(\mathrm{K}^{+}\right.$and $\left.\mathrm{Cl}^{-}\right)$.

The apparent conclusion from this analysis is that many of the genes affecting motor neuron excitability share expression patterns, where the majority having an excitatory effect are up-regulated in late stages of the injury response (21 and 60 days post injury) falling into clusters 6-10 while the majority of the genes relating to inhibition are down-regulated in the late phases of the response falling into cluster profiles 1-4 and 12.

\section{Transcriptional control of gene clusters}

The distinct expression pattern shared by the genes of each consensus cluster and their associated ontology terms suggest a common regulatory control of each gene cluster. This possibility was examined by matching transcription factor binding sites with core promoter sequences of the genes associated with each cluster using ASAP [38] with motifs obtained from the TRANSFAC database. This database is at present not fully annotated for all know transcriptions factors, and at the time of writing TRANSFAC contained motifs for 6 of the 34 transcription factors encoded by genes identified in the present study as differentially expressed (SP1, E2F1, FOXO1, ATF3, ATF4, MYC). Based on over-representation analysis of their binding sites we find that the expression pattern for the genes of these six transcription factors correlate nicely with the time profiles of their putative target gene clusters. The genes of SP1 (Spl), E2F1 (E2fl) and FOXO1 (Foxo1) all belong to time profile 7 with a common up-regulation throughout the injury response, but have different targets clusters.

The binding sites of SP1 are over-represented in profiles 6-11 suggesting a positive regulation by this transcription factor of the genes associated with these profiles. The binding sites of E2F1 are over-represented in gene clusters 1, 4 and 6-12 suggesting the interaction of this transcription factor with other proteins to focus its regulatory effect to fewer transcript targets. Interestingly the non-specific general activator proteins SP1 and E2F1 have been shown to interact to promote transcription [45] and in motor neurons they can drive the transcription of the motor neuron specific transcription factor HB9 [46]. Their common binding site over-representation in cluster profiles 6-11 therefore suggest that they participate in a general activation of transcription of their associated genes.

The binding sites of FOXO1 are over-represented in cluster profiles 1-4 plus 12 and it thus seems to have a suppressive effect. The fork-head transcription factor FOXO1 has not previously been associated with the spinal cord, but it has been found in other parts of the developing and adult brain [47].

ATF3 and ATF4 both belong to the mammalian activation transcription factor/cAMP responsive element-binding (CREB) protein family of transcription factors and they have both been associated with trauma relating to the spinal cord. ATF3 has been shown to be up-regulated in motor and sensory neurons subject to axotomy [48] as well as in spinal neurons post injury [49]. The up-regulation of ATF4 has been associated with ischemia of both brain and spinal cord [49]. The genes of these two transcription factors follow different expression profiles. The gene of ATF4 (Atf4) belongs to expression profile 10 and thus is subject to an initial repression followed by a late up-regulation. The binding site of ATF4 is over-represented in time profile 5 suggesting that its late expression is suppressing the genes of cluster 5 as they return to control levels after the initial up-regulation. Since the gene of ATF3 (Atf3) belongs to cluster 5 it therefore seems to be subject to the repression of ATF4. The early up-regulation of the gene coding for ATF3 (Atf3) on the other hand might correlate with initial up-regulation of expression in clusters with over-representation of its binding site, clusters 7, 8, and 9. The binding site of ATF3 is also over-represented in gene clusters 11 and 12 with early downregulation of expression, thus ATF3 must work together with other transcription factors to explain this apparent opposing effect on the expression of its target genes.

MYC, a member of the myc-family of transcription factors, is a complex regulator of general transcriptional activation [50] and has been associated with immediate early injury response of neurons in the spinal cord [51]. The gene of this transcription factor belongs to cluster 
profile 5 together with the gene of ATF3. The binding site for MYC is over-represented in gene clusters 6-8 and 1011 suggesting a role in the early regulation of the genes in these clusters.

The motif analysis could not be conducted on the full set of transcription factors with differential expression of their associated genes as not all of these were contained in TRANSFAC, which otherwise would have enabled us to make a more complete estimate of the regulatory network underlying the expression patterns observed in the motor neurons as a response to the injury. Apart from the six transcription factors with know motifs, we note that several of the other transcription factors with differential expression of their associated genes have been implicated with central nervous system development or its response to trauma. These include the down-regulated genes of the transcription factors E2F5 (E2f5) [51], GT2D1 (Gtf2ird1, synonymous with BEN) [52] and NFIA (Nfia) [53] as well as the up-regulated genes of transcription factors PBX3 (Pbx3) [54] and NRF2F (Nrf2f) [55]. In particular OLIG1 and NKX6-2 have been implicated in motor neuron differentiation early in development [56-58]. The down-regulation of their genes (Olig1 and Nkx6-2) compared to the un-injured state also suggests a role for these in the maintenance of normal motor neuron function and identity.

There is growing supporting evidence for a model where the pattern of neurogenesis is achieved through a mechanism of controlled repression of transcription upon a background of non-specific transcriptional activation $[46,59,60]$. It therefore seems like the developing nervous system is subject to general transcriptional activation by non-specific general-activator transcription factors while the cell specific processes are directed by controlled inhibition of transcription. If this mechanism applies to the adult organism, and in particular to the injury response of motor neurons observed in the present case, the down-regulation of a suppressor transcription factor could have the same effect as the up-regulation of a transcription factor enhancer targeting the same genes, i.e. induce transcription. The expression pattern of the genes coding for SP1, E2F1 and MYC together with their broad cluster targets suggest that these un-specific activators of transcription enhance the general transcriptional capacity of the motor neurons, while the expression pattern of genes coding for other more specific regulators of transcription like OLIG1 or NKX6-2 could function to shape the response by relief or activation of targeted suppression of specific sets of genes, supporting the hypothesis of suppressor mediated transcriptional specificity.

The observed combination of up- and down-regulated transcription factors therefore suggests a redirection of the transcriptional program, where the transcription factors of clusters 1-4 and 12 must be involved in the main- tenance of normal motor neuron function and their down-regulation together with the up-regulated transcription factors of cluster profiles 5-10 suggest a dynamic transition to a new transcriptional state. It is also clear from our analysis that these transitions through different transcriptional states across time are mediated by the interactions of several transcription factors.

\section{Conclusion}

The present study expand our previous work on the late transcriptional response of motor neurons following spinal cord injury by adding data from the early phase, resulting in a data set comprising days $0,2,7,21$ and 60 post injury. The consensus clustering with the subsequent ontology analysis enabled us to identify distinct expression time profiles from which we can describe the biological processes as they progress over time and correlate them to the pathophysiologal development of spinal cord injury. Extracting genes directly relating to motor neuron excitability further focus the analysis towards changes associated with injury-induced hyper-reflexia. The cluster identity of these genes in complement with the over-representation analysis on GO terms and transcription factor binding sites indicate some general mechanism of how the motor neurons regulate their membrane excitability as a response to the injury.

Our analysis clearly suggests that the transcriptional response of the motor neurons to injury is complex, and that the observed increased excitability is the result of many interacting factors. This study therefore provides a first step towards an understanding of the correlation between the transcriptional regulation in an individual cell population and the physiological state of a biologically complex system. In this light it therefore seems unlikely that the suppression of a single gene or protein relating to ion channels or receptors will have a significant effect in reducing motor neuron excitability to alleviate injury-induced spasticity. We therefore suggest an alternative approach, where the manipulation of the transcriptional regulators such as the identified transcription factors could be used to alter the transcriptional response to prevent the motor neurons from entering a state of hyper-excitability.

\section{Methods}

\section{Spinal cord preparation}

All handling of animals was approved by the Danish Animal Experiments Inspectorate. The handling and experimental procedures of the animals were conducted at University of Copenhagen (Denmark) and the isolated spinal cord tissue was further processed at Karolinska Institutet in Stockholm (Sweden).

Adult male Wistar rats (325-480 g) were used in this study. The animals used for microarray hybridization 
were separated into five groups: controls of un-injured animals (Control; $n=4)$, spinalized for 2 days (Spi-2; $n=$ 6), spinalized for 7 days (Spi-7; $n=5$ ), spinalized for 21 days $($ Spi-21; $\mathrm{n}=8)$ and spinalized for 60 days $($ Spi-60, $\mathrm{n}=$ 8). The Spi-21 and Spi-60 samples were obtained from a previous study [32] and the remaining samples were produced as described therein. In short, laminectomy was performed on animals under anesthesia between the lumbar L2 and L3 vertebras and injury was inflicted on the spinal cord by removing 1-2 $\mathrm{mm}$ tissue at the sacral S2 segment. After spinalization, the wound was closed suturing muscles, muscle fascia and skin separately. Care was taken to relieve pain post-operatively. Until termination of the experiment the welfare of the rats were routinely checked (e.g. for signs of infections, motor loss or bladder dysfunction) and rats that showed signs of distress were immediately euthanized. Since the spinal cord injury was inflicted at the S2 level only the motor and sensory functions of the tail were affected leaving the bladder, bowel as well as hind limb functions intact. Motor neurons were labeled in vivo with Fluoro-Gold (Fluorochrome) as described in [25]. At the day of termination animals were anesthetized with pentobarbital (initially $20 \mathrm{mg} / \mathrm{kg}$ and then $5 \mathrm{mg} / \mathrm{kg}$ every 30 minute,

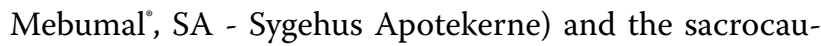
dal spinal cords were removed, snap-frozen in liquid nitrogen and stored at $-80^{\circ} \mathrm{C}$ until further processed.

\section{Motor neuron extraction and microarray preparation}

Fluoro-Gold labeled motor neurons (at the S3-S4 level) were laser microdissected and their RNA extracted and amplified as previously described [32]. In short, retrogradely Fluoro-Gold labeled motor neurons were isolated from 10 thin spinal cord cryosections using the Leica AS laser microdissection system (Leica Microsystems) at room temperature. From each rat the total RNA was isolated from 70-200 laser microdissected motor neurons using the PicoPure ${ }^{\text {mix }}$ RNA Isolation Kit (Arcturus) and the messenger RNA (mRNA) fraction was amplified in a two round T7 linear amplification process using the RiboAmp $^{\text {tu }}$ HS RNA Amplification Kit (Arcturus). The complementary DNA (cDNA) product from the $2^{\text {nd }}$ round of the amplification process was used to generate biotinlabelled antisense RNA (aRNA) (GeneChip Expression 3'-Amplification Reagents for IVT Labeling, Affymetrix). The integrity and concentration of the amplified and biotinylated aRNA was assessed on an Agilent RNA chip with the Agilent 2100 bioanalyzer (Agilent Technologies) both before and after fragmentation. Only samples of good integrity were further used and $15 \mu \mathrm{g}$ of the fragmented samples were hybridized to GeneChip Rat Genome 2302.0 Arrays (RAT230_2 chip, Affymetrix) and subsequently scanned. Each array always originated from a single animal. The Agilent analysis and microarray hybridizations were conducted at the Affymetrix core facility at Novum (Bioinformatics and Expression Analysis core facility, Department of Biosciences and Nutrition, Karolinska Institutet, Huddinge, Sweden).

\section{Microarray preprocessing}

The microarray normalization and the analysis for detection of significantly differentially expressed genes was adopted from Ryge and colleagues [34]. We used the Affymetrix probe sets verbatim, but discarded those not included in the Ensembl database for the RAT230 2 chip prior to the statistical analysis, reducing the set of probes from 31,099 to 12,919 . The microarrays were then background compensated, normalized and RMA (Robust Multi-array Average) expression summaries were calculated [34,61]. Additional background compensation was carried out on the expression summaries as described in [25]. Inspection of the normalized distributions showed that all microarray RMA profiles followed the average distribution throughout the intensity range, validating the microarray pre-processing steps (Additional file 2). The RMA expression summaries together with the raw CEL files for all microarrays were submitted to the Gene Expression Omnibus (GEO; http://www.ncbi.nlm. nih.gov/geo/) hosted by the National Center for Biotechnology Information and can be accesses under accession number GSE19701.

\section{Differentially expressed genes}

To determine the significantly differentially expressed genes across the five time points adjusted ANOVA analysis' were performed using three different statistical procedures: Cyber-T, limma and SAM (described in [62-65]). Each time point was treated as a separate "biological condition", in essence identifying genes violating the null hypothesis of equal mean across all conditions. The resulting test statistics of all three procedures were then used to create a conglomerate ranking of each gene reflecting their degree of significance across all three tests as described in [34]. For the purpose of clustering a FDR cut-off of 0.02 was chosen classifying 3,708 out of 12,919 genes as differentially expressed. To compensate for multiple testing the p-values of Cyber-T and limma were converted to FDRs using the approach of Allison and colleagues [66], whereas the FDRs of SAM are based on a methodology of permutation and re-sampling of the data (i.e. these FDRs are output from the SAM analysis directly).

\section{Consensus Cluster analysis}

ClusterLustre, a robust consensus clustering method, was used to group the set of differentially expressed genes into clusters of reliably classified gene expression patterns [35]. To avoid clustering according to magnitude but rather on common patterns of expression, the expression 
level of each transcript were normalized prior to clustering:

$$
x_{n m}=\frac{y_{n m}-\bar{y}_{n}}{\sqrt{\sum_{m=1}^{M}\left(y_{n m}-\bar{y}_{n}\right)^{2}}}
$$

for gene $n=1, \ldots, N$ and microarray $m=1, \ldots, N$. Here $y_{n m}$ represents the RMA expression value of transcript $n$ on chip $m$ and $\bar{y}_{n}$ the average expression level of transcript $n$ across all $M$ microarrays. The denominator is used to confine the expression variance to the interval $[-1,1]$. The consensus clustering algorithm aims at producing robust clustering results by averaging over multiple clustering runs such that the sensitivity to settings and initialization are diminished. The algorithm works as follows (for more details see [35]). Initially, 30 scans with k-means in the interval $\mathrm{k}=6 \ldots 14$, leading to a total of $9 * 30=270$ clustering runs are performed. From these a co-occurrence matrix reflecting the pair wise probability of transcripts falling in the same cluster is made. The resulting cooccurrence matrix thus describes the pair wise empirical probability of transcripts falling in the same cluster throughout the 270 clustering runs. In the final step, one minus the co-occurrence matrix (= the dissimilarity matrix) is used as input to hierarchical clustering. This gives a robust clustering because genes that fall in the same cluster across most cluster runs will have a small dissimilarity and thus be grouped together, whereas this is not the case for genes that infrequently by chance are in the same cluster. So, consensus clustering gives clearer clusters than a direct application of hierarchical clustering on the data [35].

In the present study, we found 12 consensus clusters to be optimal in terms of representing distinct expression time profiles (each containing approximately 150-600 genes). A full list of the differentially expressed genes grouped according to their cluster ID is provided in Additional file 1 .

\section{Over-representation analysis on identified gene clusters Ontologies}

The DAVID online ontology-cluster tool $[67,68]$ was used on each of the 12 identified gene clusters to identity groups of over-represented ontologies sharing a gene overlap of minimum $70 \%$, from which a significant representative term was extracted. Ontology clusters were deemed significant if they contained ontologies with $\mathrm{p}$ values below 0.03 .

\section{Transcription factors}

To identify transcription factors that may be involved in regulation of the identified gene clusters we performed motif analysis on each cluster looking for transcription factor binding sites in the core promoter sequences of their constituent genes. For this analysis we obtained the sequences from the Ensembl database of $1000 \mathrm{bp}$ upstream and $200 \mathrm{bp}$ downstream of the coding region of all differentially expressed genes using biomaRt. Each set of promoter sequences pertaining to a distinct time profile was analyzed for over- and under-represented transcription factor binding sites using ASAP [38] with position weight matrices (PWM) obtained from JASPAR [39] or TRANSFAC [40]. ASAP searches the set of promoter sequences using a transcription factor motif (i.e. PWM) and calculates the number of times a given binding site appears, producing a $\mathrm{Z}$ score reflecting the likelihood of its over-representation (positive) or underrepresentation (negative) compared to the amount of times it appears in a pre-defined background. In the present case the 3,708 differentially expressed genes were used as background for each cluster.

We note that the presented findings resting on this methodology are based on predictions and that more than one transcription factors may bind to the same target. This means that over-representation of a certain binding site for one transcription factor in a gene promoter region may not always predict the binding of the exact same factor.

\section{Differentially expressed genes relating to motor neuron excitability}

Significantly differentially expressed genes relating to motor neuron excitability were extracted along with their corresponding cluster ID, belonging to the same categories of ion channels and neurotransmitter receptors as described in [25]. In particular genes relating to $\mathrm{Ca}^{2+}$, $\mathrm{Na}^{+}, \mathrm{Cl}^{-}$and $\mathrm{K}^{+}$channels as well as genes relating to glutamatergic, GABAergic, glycinergic, cholinergic, serotonergic, adrenergic and dopaminergic receptors were identified and are shown in Table $3 . \mathrm{Ca}^{2+}$ binding genes relating to calmodulin and $\mathrm{IP}_{3}$ were also included in this table.

\section{Software}

The microarray analysis was done using $\mathrm{R}$ http://www.rproject.org/ and Bioconductor http://bioconductor.org. Cyber-T source code was obtained from the website http://cybert.microarray.ics.uci.edu/. Open source software was used for clustering (ClustreLustre, http:// eivind.imm.dtu.dk/staff/winther/software.html) and promoter analysis (ASAP, http://asap.binf.ku.dk/Asap/ Home.html). Separate scripts for integration of $\mathrm{R}$ and bioconductor data formats with these programs were developed and are included as supplementary material (Additional files 3 and 4). 


\section{Additional material}

\section{Additional file 1 Table containing all differentially expressed genes} and their consensus cluster ID.

Additional file 2 Figure showing Validation of normalization. Distributions of linear-normalized probe intensities (perfect match, PM) and RMA expression summaries. Quantile-quantile plots B and D illustrate the variation of the distribution tails of the distributions plotted in $A$ and $C$. The nice overlap of both PM and RMA distributions validates the normalization procedure and the quality of the data obtained from different animals at different time points following spinal cord injury. A. Plot of linear-quantilenormalized PM distributions of each microarray. B. Quantile-quantile plot: linear-quantile-normalized PM distributions (shown in A) plotted against the average PM distribution. $C$. Distributions of normalized RMA expression summaries, based on PM values from $A$ and $B$, which has undergone further quantile normalization. D. Quantile-quantile plot: normalized RMA expression summary distributions (shown in C) plotted against the average RMA distribution.

Additional file $\mathbf{3} \mathbf{R}$ scripts source file. An R source file containing scripts for probe annotation, export and import of expression values to and from ClustreLustre and cluster plots, extraction of sequences using biomaRt for ASAP analysis.

Additional file 4 R scripts example. Text file illustrating an example of how to run scripts (details on ClustreLustre and ASAP usage is described in original papers)

\section{Authors' contributions}

JR participated in the design of the study, participated in data acquisition, performed data analysis and wrote the manuscript. OW formed strategy for and participated in cluster analysis, participated in promoter analysis and participated in manuscript revision. JW performed the animal surgeries and participated in data acquisition. ACW performed the molecular work and participated in data acquisition. AS participated in promoter analysis and participated in manuscript revision. $\mathrm{HH}$ participated in design of study and participated in writing the manuscript. OK participated in the design and coordination of the study and wrote the manuscript. All authors read and approved the final manuscript.

\section{Acknowledgements}

We especially thank Lillian Grøndahl for her great assistance during surgeries and in the following handling of spinalized animals.

\section{Author Details}

${ }^{1}$ Mammalian Locomotor Laboratory, Department of Neuroscience, Karolinska Institutet, Retzius väg 8, 17177 Stockholm, Sweden, 2Bioinformatics Centre Department of Biology and Biotech Research and Innovation Centre (BRIC), University of Copenhagen, Ole Maaloes Vej 5, 2200 Copenhagen, Denmark, ${ }^{3}$ DTU Informatics, Technical University of Denmark, 2800 Lyngby, Denmark, University of Copenhagen, Ole Maaloes Vej 5, 2200 Copenhagen, Denmark and ${ }^{4}$ Department of Neuroscience and Pharmacology, University of Copenhagen, Blegdamsvej 3, 2200 Copenhagen, Denmark

Received: 4 March 2010 Accepted: 9 June 2010 Published: 9 June 2010

\section{References}

1. Rossignol S, Schwab M, Schwartz M, Fehlings MG: Spinal cord injury: time to move? JNeurosci 2007, 27(44):11782-11792.

2. Little JW, Ditunno JF Jr, Stiens SA, Harris RM: Incomplete spinal cord injury: neuronal mechanisms of motor recovery and hyperreflexia. Archives of physical medicine and rehabilitation 1999, 80(5):587-599.

3. Hultborn $\mathrm{H}$ : Changes in neuronal properties and spinal reflexes during development of spasticity following spinal cord lesions and stroke: studies in animal models and patients. J Rehabil Med 2003, 41(Suppl):46-55.

4. Frigon A, Rossignol S: Functional plasticity following spinal cord lesions. Progress in brain research 2006, 157:231-260

5. Ritz LA, Friedman RM, Rhoton EL, Sparkes ML, Vierck CJ Jr: Lesions of cat sacrocaudal spinal cord: a minimally disruptive model of injury. Journal of neurotrauma 1992, 9(3):219-230.
6. Bennett DJ, Gorassini M, Fouad K, Sanelli L, Han Y, Cheng J: Spasticity in rats with sacral spinal cord injury. Journal of neurotrauma 1999, 16(1):69-84.

7. Nielsen JB, Crone C, Hultborn H: The spinal pathophysiology of spasticity--from a basic science point of view. Acta physiologica (Oxford, England) 2007, 189(2):171-180

8. Biering-Sorensen F, Nielsen JB, Klinge K: Spasticity-assessment: a review. Spinal Cord 2006, 44(12):708-722

9. Crone $\mathrm{C}$, Hultborn $\mathrm{H}$, Kiehn O, Mazieres L, Wigstrom H: Maintained changes in motoneuronal excitability by short-lasting synaptic inputs in the decerebrate cat. The Journal of physiology 1988, 405:321-343.

10. Kiehn $\mathrm{O}$, Eken $\mathrm{T}$ : Functional role of plateau potentials in vertebrate motor neurons. Current opinion in neurobiology 1998, 8(6):746-752.

11. Hultborn $\mathrm{H}$ : Plateau potentials and their role in regulating motoneuronal firing. Progress in brain research 1999, 123:39-48.

12. Heckmann CJ, Gorassini MA, Bennett DJ: Persistent inward currents in motoneuron dendrites: implications for motor output. Muscle \& nerve 2005, 31(2):135-156.

13. Schwindt $P$, Crill WE: A persistent negative resistance in cat lumbar motoneurons. Brain Res 1977, 120(1):173-178.

14. Schwindt PC, Crill WE: Properties of a persistent inward current in normal and TEA-injected motoneurons. Journal of neurophysiology 1980, 43(6):1700-1724

15. Hultborn H, Kiehn O: Neuromodulation of vertebrate motor neuron membrane properties. Current opinion in neurobiology 1992, 2(6):770-775.

16. Conway BA, Hultborn $\mathrm{H}$, Kiehn O, Mintz I: Plateau potentials in alphamotoneurones induced by intravenous injection of L-dopa and clonidine in the spinal cat. The Journal of physiology 1988, 405:369-384

17. Hounsgaard J, Hultborn $H$, Jespersen B, Kiehn O: Bistability of alphamotoneurones in the decerebrate cat and in the acute spinal cat after intravenous 5-hydroxytryptophan. The Journal of physiology 1988, 405:345-367.

18. Hounsgaard J, Kiehn O: Serotonin-induced bistability of turtle motoneurones caused by a nifedipine-sensitive calcium plateau potential. The Journal of physiology 1989, 414:265-282.

19. Delgado-Lezama R, Perrier JF, Nedergaard S, Svirskis G, Hounsgaard J: Metabotropic synaptic regulation of intrinsic response properties of turtle spinal motoneurones. The Journal of physiology 1997, 504(Pt 1):97-102.

20. Lee $\mathrm{RH}, \mathrm{Heckman} \mathrm{CJ}$ : Enhancement of bistability in spinal motoneurons in vivo by the noradrenergic alpha1 agonist methoxamine. Journal of neurophysiology 1999, 81(5):2164-2174

21. Alaburda A, Hounsgaard J: Metabotropic modulation of motoneurons by scratch-like spinal network activity. J Neurosci 2003, 23(25):8625-8629.

22. Eken $\mathrm{T}$, Hultborn $\mathrm{H}$, Kiehn $\mathrm{O}$ : Possible functions of transmittercontrolled plateau potentials in alpha motoneurones. Progress in brain research 1989, 80:257-267. discussion 239-242

23. Bennett DJ, Li Y, Harvey PJ, Gorassini M: Evidence for plateau potentials in tail motoneurons of awake chronic spinal rats with spasticity. Journal of neurophysiology 2001, 86(4):1972-1982

24. Bennett DJ, Li Y, Siu M: Plateau potentials in sacrocaudal motoneurons of chronic spinal rats, recorded in vitro. Journal of neurophysiology 2001, 86(4):1955-1971.

25. Wienecke J, Westerdahl AC, Hultborn H, Kiehn O, Ryge J: Global gene expression analysis of rodent motor neurons following spinal cord injury associates molecular mechanisms with development of postinjury spasticity. Journal of neurophysiology 2010, 103(2):761-778.

26. Diaz E, Ge Y, Yang YH, Loh KC, Serafini TA, Okazaki Y, Hayashizaki Y, Speed TP, Ngai J, Scheiffele P: Molecular analysis of gene expression in the developing pontocerebellar projection system. Neuron 2002, 36(3):417-434

27. Arlotta P, Molyneaux BJ, Chen J, Inoue J, Kominami R, Macklis JD: Neuronal subtype-specific genes that control corticospinal motor neuron development in vivo. Neuron 2005, 45(2):207-221.

28. David H, Hofmann G, Oliveira AP, Jarmer H, Nielsen J: Metabolic network driven analysis of genome-wide transcription data from Aspergillus nidulans. Genome Biol 2006, 7(11):R108.

29. Regenberg B, Grotkjaer T, Winther O, Fausboll A, Akesson M, Bro C, Hansen LK, Brunak S, Nielsen J: Growth-rate regulated genes have profound 
impact on interpretation of transcriptome profiling in Saccharomyces cerevisiae. Genome Biol 2006, 7(11):R107.

30. Ramsey SA, Klemm SL, Zak DE, Kennedy KA, Thorsson V, Li B, Gilchrist M, Gold ES, Johnson CD, Litvak V, et al: Uncovering a macrophage transcriptional program by integrating evidence from motif scanning and expression dynamics. PLoS computational biology 2008, 4(3):e1000021.

31. Segal E, Shapira M, Regev A, Pe'er D, Botstein D, Koller D, Friedman N: Module networks: identifying regulatory modules and their conditionspecific regulators from gene expression data. Nat Genet 2003, 34(2):166-176.

32. Wienecke J, Westerdahl AC, Hultborn H, Kiehn O, Ryge J: Global gene expression analysis of rodent motor neurons following spinal cord injury associate molecular mechanisms with development of postinjury spasticity. Journal of neurophysiology 2009.

33. Bennett DJ, Sanelli L, Cooke CL, Harvey PJ, Gorassini MA: Spastic longlasting reflexes in the awake rat after sacral spinal cord injury. Journal of neurophysiology 2004, 91(5):2247-2258.

34. Ryge J, Westerdahl AC, Alstrom P, Kiehn O: Gene expression profiling of two distinct neuronal populations in the rodent spinal cord. PLOS ONE 2008, 3(10):e3415.

35. Grotkjaer T, Winther O, Regenberg B, Nielsen J, Hansen LK: b $>$ Robust multi-scale clustering of large DNA microarray datasets with the consensus algorithm. Bioinformatics 2006, 22(1):58-67.

36. Khatri P, Draghici S: Ontological analysis of gene expression data: current tools, limitations, and open problems. Bioinformatics 2005, 21(18):3587-3595.

37. Wasserman WW, Sandelin A: Applied bioinformatics for the identification of regulatory elements. Nature reviews 2004, 5(4):276-287.

38. Marstrand TT, Frellsen J, Moltke I, Thiim M, Valen E, Retelska D, Krogh A: Asap: a framework for over-representation statistics for transcription factor binding sites. PLOS ONE 2008, 3(2):e1623.

39. Bryne JC, Valen E, Tang MH, Marstrand T, Winther O, da Piedade I, Krogh A, Lenhard B, Sandelin A: JASPAR, the open access database of transcription factor-binding profiles: new content and tools in the 2008 update. Nucleic acids research 2008:D102-106.

40. Matys V, Kel-Margoulis OV, Fricke E, Liebich I, Land S, Barre-Dirrie A, Reuter I, Chekmenev D, Krull M, Hornischer K, et al.: TRANSFAC and its module TRANSCompel: transcriptional gene regulation in eukaryotes. Nucleic acids research 2006:D108-110.

41. Chan CC: Inflammation: beneficial or detrimental after spinal cord injury? Recent patents on CNS drug discovery 2008, 3(3):189-199.

42. Lotan M, Schwartz M: Cross talk between the immune system and the nervous system in response to injury: implications for regeneration. Faseb J 1994, 8(13):1026-1033.

43. Velardo MJ, Burger C, Williams PR, Baker HV, Lopez MC, Mareci TH, White TE, Muzyczka N, Reier PJ: Patterns of gene expression reveal a temporally orchestrated wound healing response in the injured spinal cord. J Neurosci 2004, 24(39):8562-8576.

44. Cullheim S, Thams S: The microglial networks of the brain and their role in neuronal network plasticity after lesion. Brain research reviews 2007 55(1):89-96.

45. Karlseder J, Rotheneder H, Wintersberger E: Interaction of Sp1 with the growth- and cell cycle-regulated transcription factor E2F. Molecular and cellular biology 1996, 16(4):1659-1667.

46. Lee SK, Jurata LW, Funahashi J, Ruiz EC, Pfaff SL: Analysis of embryonic motoneuron gene regulation: derepression of general activators function in concert with enhancer factors. Development (Cambridge, England) 2004, 131(14):3295-3306

47. Hoekman MF, Jacobs FM, Smidt MP, Burbach JP: Spatial and temporal expression of FoxO transcription factors in the developing and adult murine brain. Gene Expr Patterns 2006, 6(2):134-140.

48. Tsujino H, Kondo E, Fukuoka T, Dai Y, Tokunaga A, Miki K, Yonenobu K, Ochi T, Noguchi K: Activating transcription factor 3 (ATF3) induction by axotomy in sensory and motoneurons: A novel neuronal marker of nerve injury. Molecular and cellular neurosciences 2000, 15(2):170-182.

49. Huang WL, George KJ, Ibba V, Liu MC, Averill S, Quartu M, Hamlyn PJ, Priestley JV: The characteristics of neuronal injury in a static compression model of spinal cord injury in adult rats. The European journal of neuroscience 2007, 25(2):362-372.

50. Varlakhanova NV, Knoepfler PS: Acting Locally and Globally: Myc's EverExpanding Roles on Chromatin. Cancer research 2009, 69(19):7487-90.
51. Di Giovanni S, Knoblach SM, Brandoli C, Aden SA, Hoffman EP, Faden Al: Gene profiling in spinal cord injury shows role of cell cycle in neuronal death. Annals of neurology 2003, 53(4):454-468.

52. Dillon AK, Fujita SC, Matise MP, Jarjour AA, Kennedy TE, Kollmus H, Arnold $\mathrm{HH}$, Weiner JA, Sanes JR, Kaprielian Z: Molecular control of spinal accessory motor neuron/axon development in the mouse spinal cord. J Neurosci 2005, 25(44):10119-10130.

53. Mason S, Piper M, Gronostajski RM, Richards LJ: Nuclear factor one transcription factors in CNS development. Molecular neurobiology 2009, 39(1):10-23

54. Rottkamp CA, Lobur KJ, Wladyka CL, Lucky AK, O'Gorman S: Pbx3 is required for normal locomotion and dorsal horn development. Developmental biology 2008, 314(1):23-39.

55. Kim BJ, Takamoto N, Yan J, Tsai SY, Tsai MJ: Chicken Ovalbumin Upstream Promoter-Transcription Factor II (COUP-TFII) regulates growth and patterning of the postnatal mouse cerebellum. Developmental biology 2009, 326(2):378-391.

56. Zhou Q, Anderson DJ: The bHLH transcription factors OLIG2 and OLIG1 couple neuronal and glial subtype specification. Cell 2002, 109(1):61-73.

57. Lu Q, Sun T, Zhu Z, Ma N, Garcia M, Stiles CD, Rowitch DH: Common developmental requirement for Olig function indicates a motor neuron/oligodendrocyte connection. Cell 2002, 109(1):75-86.

58. Pattyn A, Vallstedt A, Dias JM, Sander M, Ericson J: Complementary roles for Nkx6 and Nkx2 class proteins in the establishment of motoneuron identity in the hindbrain. Development (Cambridge, England) 2003, 130(17):4149-4159.

59. Muhr J, Andersson E, Persson M, Jessell TM, Ericson J: Groucho-mediated transcriptional repression establishes progenitor cell pattern and neuronal fate in the ventral neural tube. Cell 2001, 104(6):861-873.

60. Vallstedt A, Muhr J, Pattyn A, Pierani A, Mendelsohn M, Sander M, Jessell TM, Ericson J: Different levels of repressor activity assign redundant and specific roles to Nkx6 genes in motor neuron and interneuron specification. Neuron 2001, 31(5):743-755.

61. Irizarry RA, Bolstad BM, Collin F, Cope LM, Hobbs B, Speed TP: Summaries of Affymetrix GeneChip probe level data. Nucleic acids research 2003, 31(4):-e15.

62. Baldi P, Long AD: A Bayesian framework for the analysis of microarray expression data: regularized $\mathrm{t}$-test and statistical inferences of gene changes. Bioinformatics 2001, 17(6):509-519.

63. Tusher VG, Tibshirani R, Chu G: Significance analysis of microarrays applied to the ionizing radiation response. Proceedings of the National Academy of Sciences of the United States of America 2001, 98(9):5116-5121.

64. Smyth GK: Linear models and empirical bayes methods for assessing differential expression in microarray experiments. Statistical applications in genetics and molecular biology [electronic resource] 2004, 3(1):Article3.

65. Wettenhall JM, Smyth GK: limmaGUI: a graphical user interface for linear modeling of microarray data. Bioinformatics 2004, 20(18):3705-3706.

66. Allison DB, Gadbury GL, Heo M, Fernandez JR, Lee C-I, Prolla TA, Weindruch R: A mixture model approach for the analysis of microarray gene expression data. Computational Statistics \& Data Analysis 2002, 39:1-20.

67. Huang da W, Sherman BT, Lempicki RA: Systematic and integrative analysis of large gene lists using DAVID bioinformatics resources. Nature protocols 2009, 4(1):44-57.

68. Dennis G Jr, Sherman BT, Hosack DA, Yang J, Gao W, Lane HC, Lempicki RA: DAVID: Database for Annotation, Visualization, and Integrated Discovery. Genome Biol 2003, 4(5):P3.

doi: $10.1186 / 1471-2164-11-365$

Cite this article as: Ryge et al., Transcriptional regulation of gene expression clusters in motor neurons following spinal cord injury BMC Genomics 2010, 11:365 